\title{
Persistent Organic Pollutants in Human Breast Milk and Associations with Maternal Thyroid Hormone Homeostasis
}

Zhong-Min Li $i^{*,+,}$, Michael Albrecht ${ }^{\#}$,Hermann Fromme ${ }^{\S, \|}$, Karl-Werner Schramm ${ }^{\dagger, \nabla}$, Meri De Angelis ${ }^{\dagger}$

${ }^{\dagger}$ Helmholtz Zentrum München-German Research Center for Environmental Health (GmbH), Molecular EXposomics, Ingolstädter Landstr. 1, 85764 Neuherberg, Germany

${ }^{\ddagger}$ School of Life Sciences Weihenstephan (Nutrition), Technische Universität München, 85354 Freising, Germany

\#Bavarian Health and Food Safety Authority, Department of Dioxins, Irradiation, Nitrosamines, Radioactivity, Veterinaerstr. 2, D-85764 Oberschleissheim, Germany

${ }^{\S}$ Bavarian Health and Food Safety Authority, Department of Chemical Safety and Toxicology, Pfarrstrasse 3, D-80538 Munich, Germany

"Institute and Clinic for Occupational, Social and Environmental Medicine, University Hospital, LMU Munich, Ziemssenstrasse 1, D-80336, Munich, Germany

$\nabla^{D}$ Department für Biowissenschaftliche Grundlagen, Technische Universität München,

Weihenstephaner Steig 23, 85350 Freising, Germany 


\section{Correspondence:}

Zhong-Min Li, Helmholtz Zentrum München-German Research Center for Environmental Health (GmbH), Molecular EXposomics, Ingolstädter Landstr. 1, 85764 Neuherberg, Germany.

E-mail:lizmgs@hotmail.com,zhong-min.li@helmholtz-muenchen.de

Tel. +49893187 2932, Fax. +498931873371 


\section{Table of Content}

Supporting method

Table S1 Optimized MS/MS parameters for thyroid hormones. For each compound SRM iontransitions are shown as $\mathrm{m} / \mathrm{z}$ for parent ion and two product ions (for quantification and confirmation). Compound optimized values for retention time $\left(t_{R}\right)$, fragmentor $(F)$, collision energy (CE), collision acceleration voltage (CAV) and dwell times. . $\mathrm{S} 8$

Table S2 Method evaluation parameters of the optimized method for analyzing THs in human breast milk

Table S3. Concentrations of THs in human breast milk measured in this study and those reported previously (Concentrations of 3,3'- $\mathrm{T}_{2}, 3,5-\mathrm{T}_{2}, \mathrm{~T}_{1}$, and $\mathrm{T}_{1} \mathrm{AM}$ were $<\mathrm{MDL}$ )

Table S4 Descriptive statistics of PBDEs, PCBs, PCDD/Fs, and PBDD/Fs in human breast milk from LUPE study (2015-2016, Bavaria, Germany)....

Table S5 World Health Organization Toxicant Equivalent Quotient $\left(\mathrm{WHO}_{2005}\right.$-TEQ) values of PCDD/Fs, PBDD/Fs, and dioxin-like PCBs. Values are shown in $\mathrm{pg} / \mathrm{g}$ lw.

Table S6 Comparison of PBDE concentrations in human breast milk measured in different populations (ng/g lipid). Data are shown as median unless specified. S20

Table S7 Comparison of PCB concentrations in human breast milk measured in different populations (ng/g lipid). Data are shown as median unless specified

Table S8 Comparison of dioxin concentrations in human breast milk measured in different populations (pg/g lipid). Data are shown as median unless specified. S25 
Table S9 Correlations between demographic characteristics and THs in human breast milk. Comparisons were performed using Welch t-test or analysis of variance (ANOVA) S28

Table S10 Correlations between demographic characteristics and PBDEs in breast milk. Comparisons were performed using Welch t-test or analysis of variance (ANOVA). PBDE concentrations were expressed as $\mathrm{pg} / \mathrm{mL}$ milk and $\mathrm{pg} / \mathrm{g}$ lw (in the parentheses) . $\mathrm{S} 30$

Table S11 Correlations between demographic characteristics and PCBs in breast milk. Comparisons were performed using Welch t-test or analysis of variance (ANOVA). PCB concentrations were expressed as $\mathrm{pg} / \mathrm{mL}$ milk and $\mathrm{pg} / \mathrm{g}$ lw (in the parentheses) . $\mathrm{S} 36$

Table S12 Correlations between demographic characteristics and PCDD/Fs, Emono-ortho

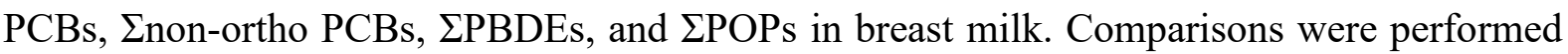
using Welch t-test or analysis of variance (ANOVA). Chemical concentrations were expressed as $\mathrm{pg} / \mathrm{mL}$ milk and $\mathrm{pg} / \mathrm{g}$ lw (in the parentheses)

Table S13 Crude associations ( $\beta$ coefficient, 95\% CI) between POPs (DF $\geq 80 \%$ ) and THs in human breast milk, single pollutant models

Table S14 Summary of the factor loadings for five factors using principal component analysis with varimax rotation S53

Table S15 Associations between exposure to tertiles of five factors from principal component analysis and TH levels based on single- and multiple-factor models. All models were adjusted for maternal age, BMI, education level, parity, country of origin, smoking, diet, and breastfeeding duration S55

Figure S1 Thyroid hormone extraction procedures in human breast milk S57 
Figure S2 Representative MRM chromatograms of THs detected in human breast milk...S58 Figure S3 Directed acyclic graph (DAG) illustrating relationships between milk POPs, THs, and covariates. S59

Figure S4 Spearman's rank correlation of biomarkers measured in human breast milk. THs were expressed in $\mathrm{ng} / \mathrm{mL}$ while POPs were expressed in $\mathrm{pg} / \mathrm{g}$ lw S60

Figure S5 Hierarchical clustering of POPs based on concentrations in 99 serum samples. The figure depicts the hierarchical structure obtained from the correlation between compounds (method: complete linkage). The horizontal red line represents the manually selected cut-off for the number of clusters S61

References. $\mathrm{S} 62$ 


\section{Supporting method}

\section{Chemicals and reagents}

$\mathrm{T}_{4}, \mathrm{~T}_{3}, \mathrm{rT}_{3}, 3,5-\mathrm{T}_{2}, \mathrm{~T}_{1}, \mathrm{~T}_{1} \mathrm{AM},{ }^{13} \mathrm{C}_{6}-\mathrm{T}_{3}$ and ${ }^{13} \mathrm{C}_{6}-\mathrm{rT}_{3}$ were from Sigma-Aldrich (St. Louis, MO, USA). 3,3'- $\mathrm{T}_{2}$ and ${ }^{13} \mathrm{C}_{6}-3,3^{\prime}-\mathrm{T}_{2}$ were from Santa Cruz Biotechnology (Dallas, Texas, USA). ${ }^{13} \mathrm{C}_{6}-\mathrm{T}_{4}$ and ${ }^{13} \mathrm{C}_{12}-\mathrm{T}_{4}$ were from Cambridge Isotope Laboratories (Andover, MA). All other reagents and solvents were of LC-MS grade. Bond Elut Plexa PCX cartridges (60 mg, 3 mL) were purchased from Agilent Technologies (Santa Clara, CA, USA).

Solvents for analysis of POPs were from high purity quality and purchased from Promochem (LGC). Materials for fat extraction were from Biotage (Isolute HM-N), Merck (sodium chloride) and Roth (anhydrous sodium sulphate). Columns for automated clean-up and fractionation with DEXTech (acidic silica, aluminium-oxide and carbon) came from LCTech. GC-columns were used from Agilent (DB-5MS, $15 \mathrm{~m} \times 0.2 \mathrm{~mm} \times 0.1 \mu \mathrm{m}$; PBDE and PBDD/F), Restek (Rtx-Dioxin2, $60 \mathrm{~m} \times 0.25 \mathrm{~mm} \times 0.25 \mu \mathrm{m}$; PCDD/F and non-orthoPCB) and SGE (HT8-PCB, $60 \mathrm{~m} \times 0.25 \mathrm{~mm} \times 0.25 \mu \mathrm{m}$; mono-ortho-PCB and ndl-PCB).

\section{Preparation of standard solutions}

A stock solution of each TH standard $\left(100 \mu \mathrm{g} / \mathrm{mL}\right.$ for $\mathrm{T}_{4}, \mathrm{~T}_{3}, \mathrm{rT}_{3}, 3,3^{\prime}-\mathrm{T}_{2}, \mathrm{~T}_{1} \mathrm{AM},{ }^{13} \mathrm{C}_{6}-\mathrm{T}_{4}$, ${ }^{13} \mathrm{C}_{6}-\mathrm{T}_{3}$ and ${ }^{13} \mathrm{C}_{6}-\mathrm{r} \mathrm{T}_{3}, 50 \mu \mathrm{g} / \mathrm{mL}$ for $3,5-\mathrm{T}_{2}, \mathrm{~T}_{1}, \mathrm{~T}_{0}$ and $\left.{ }^{13} \mathrm{C}_{12}-\mathrm{T}_{4}\right)$ was prepared using $0.1 \mathrm{~N}$ $\mathrm{NH}_{4} \mathrm{OH}$ in $\mathrm{MeOH}$ and stored at $-20{ }^{\circ} \mathrm{C}$. Calibration standard solutions $(0-150 \mathrm{ng} / \mathrm{mL}$ of each analyte and $10 \mathrm{ng} / \mathrm{mL}$ of each quantification standard) were prepared from individual stock solutions through dilution with $0.1 \%$ formic acid in $\mathrm{ACN}: \mathrm{H}_{2} \mathrm{O}(2: 8, \mathrm{v} / \mathrm{v})$. Instrumentation 
Quantification of THs was performed using an Agilent 6470 triple quadrupole tandem mass spectrometry system connected with Agilent 1290 Infinity II LC system (LC-MS/MS). The injection volume was $20 \mu \mathrm{L}$. Data acquisition and analysis were performed using Agilent MassHunter Workstation software. The mobile phases of gradient HPLC method were water (A) and $\mathrm{ACN}(\mathrm{B})$ each containing $0.1 \%$ formic acid (v/v). The column temperature was $40{ }^{\circ} \mathrm{C}$. The following gradient procedure was applied: $5 \% \mathrm{~B}$ remained for $3 \mathrm{~min}$, increased linearly to $30 \%$ B in $1 \mathrm{~min}$, ramped to $38 \%$ B gradually in $2.5 \mathrm{~min}$ and kept for $1 \mathrm{~min}$, then increased to $40 \% \mathrm{~B}$ in $1.5 \mathrm{~min}$ and kept for $1 \mathrm{~min}$, followed by an increase to $100 \% \mathrm{~B}$ in 2 min and held for $1 \mathrm{~min}$, finally returned to initial conditions in $0.2 \mathrm{~min}$. Another $3.8 \mathrm{~min}$ was allowed for re-equilibration.

\section{Sample preparation}

The optimized sample preparation procedure is shown in Figure S1. Briefly, the process contains three clean-up processes: protein precipitation, lipid elimination, and SPE extraction. For protein elimination, $\mathrm{MeOH}$ containing 3\% formic acid was incubated with breast milk. Lipids was removed by extracting with $5 \% \mathrm{CaCl}_{2}$ solution, which has been described before

1,2. Finally, the solution was further extracted with a weak cation-exchangers SPE cartridge (Bond Elut Plexa PCX).

\section{Method evaluation}

The method was evaluated by intra-day and inter-day variation, spike-recoveries, method limits of detection (LODs) and quantifications (LOQs), and matrix effects. The methods for calculating these parameters have been reported elsewhere ${ }^{1}$.

Quality assurance and quality control 
Instrumental quality control included regular injection of solvent blanks and standard solutions. The analyte identification was based on the confirmation ion and retention times compared with quantification standards. Moreover, we monitored the recovery of ${ }^{13} \mathrm{C}_{6}-\mathrm{T}_{4}$ quantification standard by spiking $0.6 \mathrm{ng}$ of ${ }^{13} \mathrm{C}_{12}-\mathrm{T}_{4}$ to each sample before injection. Compared with analytical standards in neat solution, samples that have high deviation ( $>$ $40 \%$ ) on the peak area of quantification standards or lower recovery of recovery standard $(<40 \%)$ were discarded and reanalyzed. 
Table S1 Optimized MS/MS parameters for thyroid hormones. For each compound SRM iontransitions are shown as $\mathrm{m} / \mathrm{z}$ for parent ion and two product ions (for quantification and confirmation). Compound optimized values for retention time $\left(r_{R}\right)$, fragmentor $(F)$, collision energy (CE), collision acceleration voltage (CAV) and dwell times.

\begin{tabular}{|c|c|c|c|c|c|c|c|}
\hline Compound & $\mathrm{t}_{\mathrm{R}}(\mathrm{min})$ & $\begin{array}{l}\text { Parent ion } \\
(\mathrm{m} / \mathrm{z})\end{array}$ & $\begin{array}{l}\text { Product } \\
\text { ions }(\mathrm{m} / \mathrm{z})\end{array}$ & $\mathrm{F}(\mathrm{V})$ & $\mathrm{CE}(\mathrm{V})$ & CAV (V) & $\begin{array}{l}\text { Dwell } \\
\text { (msec) }\end{array}$ \\
\hline \multicolumn{8}{|c|}{ Target compounds } \\
\hline \multirow[t]{2}{*}{$\mathrm{T}_{4}$} & 7.49 & 777.4 & $731.4(q)$ & 140 & 25 & 1 & 50 \\
\hline & & & $633.4(\mathrm{c})$ & 140 & 25 & 1 & 50 \\
\hline \multirow[t]{2}{*}{$\mathrm{T}_{3}$} & 6.70 & 651.8 & $605.6(q)$ & 120 & 25 & 1 & 50 \\
\hline & & & $507.6(\mathrm{c})$ & 120 & 25 & 1 & 50 \\
\hline \multirow[t]{2}{*}{$\mathrm{rT}_{3}$} & 6.96 & 651.8 & $605.6(q)$ & 120 & 25 & 1 & 50 \\
\hline & & & $507.6(\mathrm{c})$ & 120 & 25 & 1 & 50 \\
\hline \multirow[t]{2}{*}{$3,3^{\prime}-\mathrm{T}_{2}$} & 6.27 & 525.8 & $479.8(q)$ & 90 & 15 & 2 & 50 \\
\hline & & & 381.8 (c) & 90 & 15 & 2 & 50 \\
\hline \multirow[t]{2}{*}{$3,5-\mathrm{T}_{2}$} & 5.91 & 525.8 & $479.8(q)$ & 90 & 15 & 2 & 50 \\
\hline & & & 381.8 (c) & 90 & 15 & 2 & 50 \\
\hline \multirow[t]{2}{*}{$\mathrm{T}_{1}$} & 5.68 & 399.9 & $353.8(\mathrm{q})$ & 90 & 12 & 1 & 50 \\
\hline & & & $256(\mathrm{c})$ & 90 & 15 & 1 & 50 \\
\hline $\mathrm{T}_{1} \mathrm{AM}$ & 5.79 & 356 & $339(\mathrm{q})$ & 80 & 18 & 1 & 50 \\
\hline \multicolumn{8}{|c|}{ Quantification standards } \\
\hline $\mathrm{ML}-\mathrm{T}_{4}$ & 7.49 & 783.6 & 737.5 & 140 & 25 & 1 & 50 \\
\hline
\end{tabular}




\begin{tabular}{lccccccc}
\hline ML-T 3 & 6.70 & 657.7 & 611.6 & 120 & 25 & 1 & 50 \\
ML-rT 3 & 6.96 & 657.7 & 611.6 & 120 & 25 & 1 & 50 \\
ML-3,3'-T 2 & 6.27 & 531.8 & 485.8 & 100 & 25 & 2 & 50 \\
ML-T $_{1}$ AM & 5.79 & 362 & 345 & 104 & 12 & 1 & 50 \\
\hline
\end{tabular}

Abbreviations: $t_{R}$, retention time. $\mathrm{F}$, fragmentor. $\mathrm{CE}$, collision energy. $\mathrm{CAV}$, cell accelerator voltage. q, quantification. c, confirmation 
Table S2 Method evaluation parameters of the optimized method for analyzing THs in human breast milk.

\begin{tabular}{|c|c|c|c|c|c|c|c|}
\hline & $\mathrm{T}_{4}$ & $\mathrm{~T}_{3}$ & $\mathrm{rT}_{3}$ & $3,3^{\prime}-\mathrm{T}_{2}$ & $3,5-\mathrm{T}_{2}$ & $\mathrm{~T}_{1}$ & $\mathrm{~T}_{1} \mathrm{AM}$ \\
\hline $\operatorname{MDL}\left(\mathrm{ng} \mathrm{mL}^{-1}\right)$ & 0.01 & 0.02 & 0.01 & 0.03 & 0.08 & 0.09 & 0.13 \\
\hline MQL (ng mL $\left.{ }^{-1}\right)$ & 0.10 & 0.13 & 0.10 & 0.20 & 0.26 & 0.30 & 0.42 \\
\hline Matrix effect $(\%)$ & -9.67 & 2.13 & 4.98 & -0.37 & -1.11 & 3.26 & 14.7 \\
\hline Overall recovery $(\%)$ & 117 & 102 & 103 & 110 & 117 & 125 & 119 \\
\hline \multicolumn{8}{|c|}{ Spike-recovery $(\%), \mathrm{n}=3$} \\
\hline $1.5\left(\mathrm{ng} \mathrm{mL}^{-1}\right)$ & $102 \pm 5$ & $105 \pm 6$ & $111 \pm 3$ & $107 \pm 1$ & $112 \pm 6$ & $104 \pm 2$ & $122 \pm 3$ \\
\hline $15\left(\mathrm{ng} \mathrm{mL} L^{-1}\right)$ & $108 \pm 2$ & $106 \pm 3$ & $109 \pm 3$ & $102 \pm 2$ & $104 \pm 7$ & $112 \pm 6$ & $115 \pm 5$ \\
\hline $30\left(\mathrm{ng} \mathrm{mL} L^{-1}\right)$ & $102 \pm 5$ & $104 \pm 2$ & $107 \pm 1$ & $99.7 \pm 4.5$ & $98.4 \pm 6.1$ & $107 \pm 6$ & $115 \pm 5$ \\
\hline \multicolumn{8}{|c|}{ Intra-day variation $(\%), \mathrm{n}=3$} \\
\hline $1.5\left(\mathrm{ng} \mathrm{mL}^{-1}\right)$ & 0.47 & 4.17 & 2.53 & 0.97 & 5.06 & 1.74 & 2.25 \\
\hline $15\left(\mathrm{ng} \mathrm{mL} L^{-1}\right)$ & 1.12 & 2.33 & 2.89 & 2.07 & 6.91 & 5.18 & 4.57 \\
\hline $30\left(\mathrm{ng} \mathrm{mL}^{-1}\right)$ & 3.55 & 1.60 & 1.22 & 4.52 & 6.24 & 5.86 & 4.28 \\
\hline \multicolumn{8}{|c|}{ Inter-day variation $(\%), \mathrm{n}=6$} \\
\hline $1.5\left(\mathrm{ng} \mathrm{mL}^{-1}\right)$ & 1.56 & 3.04 & 4.10 & 4.89 & 7.71 & 6.13 & 7.18 \\
\hline $15\left(\mathrm{ng} \mathrm{mL}^{-1}\right)$ & 4.14 & 1.37 & 2.96 & 1.61 & 4.39 & 8.13 & 5.02 \\
\hline
\end{tabular}

Abbreviations: MDL: method detection limit, MQL: method quantification limit. 
Table S3. Concentrations of THs in human breast milk measured in this study and those reported previously (Concentrations of 3,3'- $\mathrm{T}_{2}, 3,5-\mathrm{T}_{2}, \mathrm{~T}_{1}$, and $\mathrm{T}_{1} \mathrm{AM}$ were $<\mathrm{MDL}$ ).

\begin{tabular}{|c|c|c|c|c|c|}
\hline $\mathrm{TT}_{4}(\mu \mathrm{g} / \mathrm{L})$ & $\mathrm{TT}_{3}(\mu \mathrm{g} / \mathrm{L})$ & $\operatorname{TrT}_{3}(\mu \mathrm{g} / \mathrm{L})$ & Method & Sample & Ref. \\
\hline $0.57 \pm 0.20$ & $0.13 \pm 0.03$ & $0.02 \pm 0.01$ & LC-MS/MS & Breast milk & This study \\
\hline $0.86 \pm 0.38$ & $0.14(0.08-0.18)$ & n.p. & RIA & Preterm breast milk & Van Wassenaer et al. ${ }^{3}$ \\
\hline $4.98 \pm 1.96$ & n.p. & n.p. & RIA & Term breast milk & Van Wassenaer et al. $^{3}$ \\
\hline \multirow[t]{3}{*}{$29.6 \pm 15.5$} & $0.35 \pm 0.20$ & n.p. & CIA & Pooled milk sample from & Zhang et al. ${ }^{4}$ \\
\hline & & & & patients with thyroid- & \\
\hline & & & & related diseases & \\
\hline
\end{tabular}

Abbreviation. n.p.: not reported. RIA, radioimmunoassay. CIA, chemiluminescence immunoassay. 
Table S4 Descriptive statistics of PBDEs, PCBs, PCDD/Fs, and PBDD/Fs in human breast milk from LUPE study (2015-2016, Bavaria, Germany).

\begin{tabular}{|c|c|c|c|c|c|c|c|c|}
\hline \multirow[t]{3}{*}{ POPs } & LOQ & $\mathrm{N}(\%)$ & Mean & Minimum & $\mathrm{Q} 1 \mathrm{pg} / \mathrm{mL}$ & Median & $\mathrm{Q} 3 \mathrm{pg} / \mathrm{mL}$ & Maximum \\
\hline & $\mathrm{pg} / \mathrm{m}$ & & $\mathrm{pg} / \mathrm{mL}$ & $\mathrm{pg} / \mathrm{mL}$ & $(\mathrm{pg} / \mathrm{g} l \mathrm{lw})$ & $\mathrm{pg} / \mathrm{mL}$ & $(\mathrm{pg} / \mathrm{g} \mathrm{lw})$ & $\mathrm{pg} / \mathrm{mL}$ \\
\hline & $\mathrm{L}$ & & (pg/g lw) & (pg/g lw) & & (pg/g lw) & & (pg/g lw) \\
\hline BDE-17 & 0.05 & $6(6)$ & $0.09(3.85)$ & $<\mathrm{LOQ}$ & $<\mathrm{LOQ}$ & $<\mathrm{LOQ}$ & $<\mathrm{LOQ}$ & $0.19(7.20)$ \\
\hline BDE-28 & 0.05 & 95 (96) & $0.95(31.7)$ & $<\mathrm{LOQ}$ & $0.49(20.2)$ & $0.81(29.3)$ & $1.21(37.7)$ & 4.44 (122) \\
\hline BDE-47 & $<1.46$ & 99 (100) & 9.34 (307) & $1.46(61.6)$ & 3.94 (136) & $5.88(204)$ & 9.69 (299) & 82.4 (2419) \\
\hline BDE-66 & 0.21 & 39 (39) & $0.14(5.69)$ & $<\mathrm{LOQ}$ & $<\mathrm{LOQ}$ & $0.09(4.20)$ & $0.19(6.20)$ & $0.49(21.9)$ \\
\hline BDE-85 & 0.09 & $18(18)$ & $0.36(10.9)$ & $<\mathrm{LOQ}$ & $0.10(4.73)$ & $0.20(9.30)$ & $0.57(17.6)$ & $1.48(27.5)$ \\
\hline BDE-99 & 0.46 & 98 (99) & $2.54(85.8)$ & $<\mathrm{LOQ}$ & $1.34(46.1)$ & $1.82(62.5)$ & $2.56(93.9)$ & $17.3(419)$ \\
\hline BDE-100 & 0.30 & 97 (98) & $2.25(73.7)$ & $<\mathrm{LOQ}$ & $0.93(31.3)$ & $1.57(54.3)$ & $2.80(92.5)$ & $12.0(364)$ \\
\hline BDE-153 & $<2.12$ & 99 (100) & $14.5(460)$ & $2.12(112)$ & $7.50(304)$ & $11.6(377)$ & $16.5(545)$ & 99.0 (1979) \\
\hline BDE-154 & 0.28 & $82(83)$ & $0.27(9.42)$ & $<\mathrm{LOQ}$ & $0.15(5.58)$ & $0.23(8.25)$ & $0.36(11.1)$ & $0.97(28.5)$ \\
\hline BDE-183 & 0.03 & 97 (98) & $1.03(33.7)$ & $<\mathrm{LOQ}$ & $0.55(19.4)$ & $0.86(28.4)$ & $1.13(42.2)$ & $6.28(182)$ \\
\hline BDE-196 & 0.09 & $89(90)$ & $0.64(22.4)$ & $<\mathrm{LOQ}$ & $0.35(12.4)$ & $0.47(16.8)$ & $0.73(24.1)$ & 4.55 (146) \\
\hline BDE-197 & $<0.31$ & 99 (100) & $2.50(83.2)$ & $0.31(19.3)$ & $1.58(53.6)$ & $2.18(73.1)$ & 3.19 (103) & $7.92(224)$ \\
\hline BDE-203 & 0.10 & $91(92)$ & $0.64(22.8)$ & $<\mathrm{LOQ}$ & $0.33(12.8)$ & 0.49 (16.7) & $0.80(24.4)$ & $3.98(265)$ \\
\hline BDE-206 & 0.11 & $86(87)$ & $4.72(176)$ & $<\mathrm{LOQ}$ & $0.58(19.1)$ & $0.86(28.9)$ & $1.65(57.3)$ & $111(3545)$ \\
\hline BDE-207 & 0.12 & 98 (99) & 4.08 (147) & $<$ LOQ & $1.18(39.7)$ & $1.75(56.3)$ & $2.82(82.0)$ & 88.7 (2842) \\
\hline BDE-208 & 0.08 & 98 (99) & $1.87(67.5)$ & $<\mathrm{LOQ}$ & $0.42(13.3)$ & 0.59 (19.3) & $1.07(34.0)$ & 48.1 (1540) \\
\hline
\end{tabular}




\begin{tabular}{|c|c|c|c|c|c|c|c|c|}
\hline BDE-209 & 0.47 & $95(96)$ & $117(4444)$ & $<\mathrm{LOQ}$ & $8.76(287)$ & $14.1(440)$ & 28.9 & 3245 \\
\hline & & & & & & & (1074) & $(104000)$ \\
\hline PCB-11 & 1.21 & 77 (78) & $3.00(105)$ & $<\mathrm{LOQ}$ & $1.56(53.6)$ & $2.66(84.5)$ & $3.96(132)$ & $15.1(438)$ \\
\hline PCB-14 & 0.56 & $0(0)$ & - & $<\mathrm{LOQ}$ & $<\mathrm{LOQ}$ & $<\mathrm{LOQ}$ & $<\mathrm{LOQ}$ & $<\mathrm{LOQ}$ \\
\hline PCB-28 & $<0.01$ & 99 (100) & $0.03(0.99)$ & $0.00(0.24)$ & $0.01(0.61)$ & $0.02(0.81)$ & $0.04(1.16)$ & $0.21(4.36)$ \\
\hline PCB-52 & $<0.01$ & 99 (100) & $0.01(0.18)$ & $0.00(0.06)$ & $0.00(0.11)$ & $0.00(0.14)$ & $0.01(0.19)$ & $0.05(1.62)$ \\
\hline PCB-77 & 0.06 & $82(83)$ & $0.10(3.55)$ & $<\mathrm{LOQ}$ & $0.06(2.37)$ & $0.08(2.97)$ & $0.12(3.83)$ & $0.38(17.2)$ \\
\hline PCB-81 & 0.03 & 19 (19) & $0.03(1.04)$ & $<\mathrm{LOQ}$ & $<\mathrm{LOQ}$ & $<\mathrm{LOQ}$ & $<\mathrm{LOQ}$ & $0.07(1.56)$ \\
\hline PCB-101 & $<0.01$ & 99 (100) & $0.01(0.37)$ & $0.00(0.10)$ & $0.01(0.18)$ & $0.01(0.25)$ & $0.01(0.34)$ & $0.19(6.20)$ \\
\hline PCB-105 & $<4.65$ & 99 (100) & $20.2(666)$ & 4.65 (177) & $11.6(459)$ & $17.7(591)$ & $23.8(785)$ & 79.0 (2067) \\
\hline PCB-114 & $<1.84$ & 99 (100) & $7.70(251)$ & $1.84(54.8)$ & $4.04(159)$ & $6.80(224)$ & $10.8(330)$ & $30.9(810)$ \\
\hline PCB-118 & $<26.7$ & 99 (100) & $119(3955)$ & $\begin{array}{l}26.7 \\
(1044)\end{array}$ & $71.5(2821)$ & $104(3619)$ & $149(4881)$ & 335 (9635) \\
\hline PCB-123 & 0.63 & $97(98)$ & $1.28(42.5)$ & $<\mathrm{LOQ}$ & $0.78(28.7)$ & 1.14 (37.6) & $1.53(55.1)$ & $3.93(110)$ \\
\hline PCB-126 & $<0.14$ & 99 (100) & $0.60(20.1)$ & $0.14(4.40)$ & $0.36(14.1)$ & $0.55(18.1)$ & $0.75(23.5)$ & $2.10(58.5)$ \\
\hline PCB-138 & $<0.11$ & 99 (100) & $0.46(15.2)$ & $0.11(3.93)$ & $0.27(10.1)$ & $0.39(14.0)$ & $0.58(18.2)$ & $1.39(35.6)$ \\
\hline PCB-153 & $<0.22$ & 99 (100) & $0.80(26.3)$ & $0.22(5.76)$ & $0.42(17.6)$ & $0.68(23.3)$ & $1.07(34.7)$ & $2.36(70.0)$ \\
\hline PCB-156 & $<18.4$ & 99 (100) & $\begin{array}{l}82.0 \\
(2668)\end{array}$ & $18.4(526)$ & $39.8(1633)$ & $\begin{array}{l}70.7 \\
(2128)\end{array}$ & $114(3573)$ & 327 (8664) \\
\hline PCB-157 & $<2.77$ & 99 (100) & $12.1(393)$ & $2.77(78.3)$ & $5.74(241)$ & $9.98(344)$ & $17.4(528)$ & $43.1(1143)$ \\
\hline PCB-167 & $<4.85$ & 99 (100) & $20.8(688)$ & 4.85 (157) & $12.0(447)$ & $18.0(662)$ & 27.5 (877) & $57.8(1481)$ \\
\hline PCB-169 & 0.03 & 98 (99) & $0.38(12.6)$ & $<\mathrm{LOQ}$ & $0.20(7.88)$ & $0.29(10.9)$ & $0.55(15.2)$ & $0.90(34.1)$ \\
\hline
\end{tabular}




\begin{tabular}{|c|c|c|c|c|c|c|c|c|}
\hline $1,2,3,4,7,8-$ & 0.02 & $1(1)$ & - & $<\mathrm{LOQ}$ & $<\mathrm{LOQ}$ & $<\mathrm{LOQ}$ & $<\mathrm{LOQ}$ & $0.17(3.33)$ \\
\hline \multicolumn{9}{|l|}{ HxBDF } \\
\hline $1,2,3,4,6,7,8$ & 0.02 & $34(34)$ & $0.31(12.2)$ & $<$ LOQ & $<$ LOQ & $<$ LOQ & $0.30(9.01)$ & $2.41(88.2)$ \\
\hline \multicolumn{9}{|l|}{ HpBDF } \\
\hline OBDF & 1.22 & $4(4)$ & 31.5 (203) & $<\mathrm{LOQ}$ & $<\mathrm{LOQ}$ & $<\mathrm{LOQ}$ & $<\mathrm{LOQ}$ & 69.1 (1337) \\
\hline \multirow[t]{2}{*}{$\Sigma$ PBDEs } & - & 99 (100) & $157(5753)$ & $9.65(511)$ & $35.2(1123)$ & 50.2 & 79.6 & 3526 \\
\hline & & & & & & (1731) & $(2727)$ & (112998) \\
\hline \multirow[t]{2}{*}{$\Sigma$ PCBs } & - & 99 (100) & $276(9090)$ & 66.9 & $159(6238)$ & $250(8322)$ & 365 & 773 (20225) \\
\hline & & & & (2222) & & & (11211) & \\
\hline$\Sigma \mathrm{PCDD} / \mathrm{Fs}$ & - & 99 (100) & $1.08(35.7)$ & $0.00(0.00)$ & $0.61(24.3)$ & $0.83(30.2)$ & $1.31(41.6)$ & 4.34 (115) \\
\hline \multirow[t]{2}{*}{$\Sigma \mathrm{PBDD} / \mathrm{Fs}$} & - & $99(100)$ & $1.58(43.2)$ & $0.00(0.00)$ & $0.00(0.00)$ & $0.14(4.53)$ & $0.38(11.5)$ & 69.88 \\
\hline & & & & & & & & (1352) \\
\hline
\end{tabular}

Abbreviations: LOQ, limit of quantification. lw, lipid weight. Q1, Q3: first and third quantile; PBDE: polybrominated diphenyl ether; PCB: polychlorinated biphenyl; PCDD/F: polychlorinated dibenzo- $p$-dioxin and furan; PBDD/F: polybrominated dibenzo- $p$-dioxin and furan. 
Table S5 World Health Organization Toxicant Equivalent Quotient $\left(\mathrm{WHO}_{2005}\right.$-TEQ) values of PCDD/Fs, PBDD/Fs, and dioxin-like PCBs. Values are shown in pg/g lw.

\begin{tabular}{|c|c|c|c|c|c|c|c|c|}
\hline & $\mathrm{N}(\%)$ & Mean & $\mathrm{SD}$ & Minimum & $\begin{array}{l}25 \% \\
\text { percentile }\end{array}$ & Median & $\begin{array}{l}75 \% \\
\text { percentil }\end{array}$ & Maximum \\
\hline PCB-77 & $82(83)$ & 0.0003 & 0.0002 & $<\mathrm{LOQ}$ & 0.0002 & 0.0003 & 0.0004 & 0.002 \\
\hline PCB-81 & $19(19)$ & 0.0002 & 0.0001 & $<\mathrm{LOQ}$ & $<\mathrm{LOQ}$ & $<\mathrm{LOQ}$ & $<\mathrm{LOQ}$ & 0.0008 \\
\hline PCB-126 & $99(100)$ & 2.01 & 0.87 & 0.44 & 1.41 & 1.81 & 2.35 & 5.85 \\
\hline PCB-169 & $98(99)$ & 0.37 & 0.19 & $<\mathrm{LOQ}$ & 0.24 & 0.33 & 0.45 & 1.02 \\
\hline PCB-105 & $99(100)$ & 0.02 & 0.01 & 0.005 & 0.01 & 0.02 & 0.02 & 0.06 \\
\hline PCB-114 & $99(100)$ & 0.008 & 0.004 & 0.002 & 0.005 & 0.007 & 0.01 & 0.02 \\
\hline PCB-118 & $99(100)$ & 0.12 & 0.05 & 0.03 & 0.08 & 0.11 & 0.15 & 0.29 \\
\hline PCB-123 & $97(98)$ & 0.001 & 0.0006 & $<\mathrm{LOQ}$ & 0.0008 & 0.001 & 0.002 & 0.003 \\
\hline PCB-156 & $99(100)$ & 0.08 & 0.04 & 0.02 & 0.05 & 0.06 & 0.11 & 0.26 \\
\hline PCB-157 & $99(100)$ & 0.01 & 0.006 & 0.002 & 0.007 & 0.01 & 0.02 & 0.03 \\
\hline PCB-167 & $99(100)$ & 0.02 & 0.009 & 0.005 & 0.01 & 0.02 & 0.03 & 0.04 \\
\hline PCB-189 & $99(100)$ & 0.008 & 0.005 & 0.001 & 0.004 & 0.007 & 0.01 & 0.04 \\
\hline 2,3,7,8-TBDD & $7(7)$ & 0.13 & 0.34 & $<\mathrm{LOQ}$ & $<\mathrm{LOQ}$ & $<\mathrm{LOQ}$ & $<\mathrm{LOQ}$ & 2.15 \\
\hline 1,2,3,7,8-PeBDD & $7(7)$ & 0.39 & 0.95 & $<\mathrm{LOQ}$ & $<\mathrm{LOQ}$ & $<\mathrm{LOQ}$ & $<\mathrm{LOQ}$ & 8.11 \\
\hline 1,2,3,4,7,8-HxBDD & $2(2)$ & 0.04 & 0.05 & $<\mathrm{LOQ}$ & $<\mathrm{LOQ}$ & $<\mathrm{LOQ}$ & $<\mathrm{LOQ}$ & 0.49 \\
\hline $1,2,3,6,7,8-\mathrm{HxBDD}$ & $3(3)$ & 0.04 & 0.04 & $<\mathrm{LOQ}$ & $<\mathrm{LOQ}$ & $<\mathrm{LOQ}$ & $<\mathrm{LOQ}$ & 0.41 \\
\hline 1,2,3,7,8,9-HxBDD & $1(1)$ & --- & --- & $<\mathrm{LOQ}$ & $<\mathrm{LOQ}$ & $<\mathrm{LOQ}$ & $<\mathrm{LOQ}$ & 0.46 \\
\hline 1,2,3,4,6,7,8-HpBDD & $38(38)$ & 0.05 & 0.04 & $<\mathrm{LOQ}$ & $<$ LOQ & $<\mathrm{LOQ}$ & 0.08 & 0.18 \\
\hline
\end{tabular}




\begin{tabular}{|c|c|c|c|c|c|c|c|c|}
\hline OBDD & $3(3)$ & 0.002 & 0.004 & $<\mathrm{LOQ}$ & $<\mathrm{LOQ}$ & $<\mathrm{LOQ}$ & $<\mathrm{LOQ}$ & 0.03 \\
\hline $2,3,7,8-\mathrm{TBDF}$ & $18(18)$ & 0.03 & 0.07 & $<\mathrm{LOQ}$ & $<\mathrm{LOQ}$ & $<\mathrm{LOQ}$ & $<\mathrm{LOQ}$ & 0.45 \\
\hline 1,2,3,7,8-PeBDF & $1(1)$ & --- & --- & $<\mathrm{LOQ}$ & $<\mathrm{LOQ}$ & $<\mathrm{LOQ}$ & $<\mathrm{LOQ}$ & 0.03 \\
\hline 2,3,4,7,8-PeBDF & $1(1)$ & --- & --- & $<\mathrm{LOQ}$ & $<\mathrm{LOQ}$ & $<\mathrm{LOQ}$ & $<\mathrm{LOQ}$ & 0.96 \\
\hline $1,2,3,4,7,8-\mathrm{HxBDF}$ & $1(1)$ & --- & --- & $<\mathrm{LOQ}$ & $<\mathrm{LOQ}$ & $<\mathrm{LOQ}$ & $<\mathrm{LOQ}$ & 0.33 \\
\hline $1,2,3,4,6,7,8-\mathrm{HpBDF}$ & $34(34)$ & 0.04 & 0.13 & $<\mathrm{LOQ}$ & $<\mathrm{LOQ}$ & $<\mathrm{LOQ}$ & 0.03 & 0.88 \\
\hline OBDF & $4(4)$ & 0.01 & 0.05 & $<\mathrm{LOQ}$ & $<\mathrm{LOQ}$ & $<\mathrm{LOQ}$ & $<\mathrm{LOQ}$ & 0.40 \\
\hline 2,3,7,8-TCDD & $30(30)$ & 0.27 & 0.40 & $<\mathrm{LOQ}$ & $<$ LOQ & $<\mathrm{LOQ}$ & 0.50 & 1.71 \\
\hline 1,2,3,7,8-PeCDD & $40(40)$ & 0.94 & 1.13 & $<\mathrm{LOQ}$ & $<$ LOQ & $<\mathrm{LOQ}$ & 1.79 & 4.33 \\
\hline 1,2,3,4,7,8-HxCDD & $87(88)$ & 0.03 & 0.06 & $<\mathrm{LOQ}$ & 0.007 & 0.009 & 0.02 & 0.52 \\
\hline 1,2,3,6,7,8-HxCDD & $24(24)$ & 0.29 & 0.20 & $<\mathrm{LOQ}$ & $<$ LOQ & $<\mathrm{LOQ}$ & $<\mathrm{LOQ}$ & 1.42 \\
\hline 1,2,3,7,8,9-HxCDD & $45(45)$ & 0.04 & 0.04 & $<\mathrm{LOQ}$ & $<$ LOQ & $<\mathrm{LOQ}$ & 0.07 & 0.17 \\
\hline 1,2,3,4,6,7,8-HpCDD & $91(92)$ & 0.03 & 0.02 & $<\mathrm{LOQ}$ & 0.02 & 0.03 & 0.04 & 0.15 \\
\hline OCDD & $98(99)$ & 0.006 & 0.004 & $<\mathrm{LOQ}$ & 0.004 & 0.005 & 0.007 & 0.02 \\
\hline $2,3,7,8-\mathrm{TCDF}$ & $9(9)$ & 0.01 & 0.02 & $<\mathrm{LOQ}$ & $<$ LOQ & $<\mathrm{LOQ}$ & $<\mathrm{LOQ}$ & 0.15 \\
\hline $1,2,3,7,8-\mathrm{PeCDF}$ & $6(6)$ & 0.003 & 0.004 & $<\mathrm{LOQ}$ & $<\mathrm{LOQ}$ & $<\mathrm{LOQ}$ & $<\mathrm{LOQ}$ & 0.03 \\
\hline 2,3,4,7,8-PeCDF & $94(95)$ & 1.20 & 0.58 & $<\mathrm{LOQ}$ & 0.85 & 1.12 & 1.54 & 3.23 \\
\hline $1,2,3,4,7,8-\mathrm{HxCDF}$ & $76(77)$ & 0.09 & 0.08 & $<\mathrm{LOQ}$ & 0.04 & 0.09 & 0.12 & 0.55 \\
\hline $1,2,3,6,7,8-\mathrm{HxCDF}$ & $80(81)$ & 0.10 & 0.09 & $<\mathrm{LOQ}$ & 0.05 & 0.09 & 0.12 & 0.79 \\
\hline $1,2,3,7,8,9-\mathrm{HxCDF}$ & $0(0)$ & --- & --- & $<\mathrm{LOQ}$ & $<\mathrm{LOQ}$ & $<\mathrm{LOQ}$ & $<\mathrm{LOQ}$ & $<\mathrm{LOQ}$ \\
\hline $2,3,4,6,7,8-\mathrm{HxCDF}$ & $40(40)$ & 0.04 & 0.08 & $<\mathrm{LOQ}$ & $<$ LOQ & $<\mathrm{LOQ}$ & 0.06 & 0.67 \\
\hline $1,2,3,4,6,7,8-\mathrm{HpCDF}$ & $60(61)$ & 0.009 & 0.02 & $<\mathrm{LOQ}$ & $<\mathrm{LOQ}$ & 0.005 & 0.01 & 0.24 \\
\hline
\end{tabular}




\begin{tabular}{|c|c|c|c|c|c|c|c|c|}
\hline 1,2,3,4,7,8,9-HpCDF & $0(0)$ & --- & --- & $<\mathrm{LOQ}$ & $<\mathrm{LOQ}$ & $<\mathrm{LOQ}$ & $<\mathrm{LOQ}$ & $<\mathrm{LOQ}$ \\
\hline OCDF & $1(1)$ & --- & --- & $<\mathrm{LOQ}$ & $<\mathrm{LOQ}$ & $<\mathrm{LOQ}$ & $<\mathrm{LOQ}$ & 0.0004 \\
\hline ¿mono-ortho PCBs & $99(100)$ & 2.38 & 0.99 & 0.54 & 1.74 & 2.20 & 2.78 & 6.57 \\
\hline ¿non-ortho PCBs & $99(100)$ & 0.27 & 0.11 & 0.06 & 0.18 & 0.25 & 0.33 & 0.60 \\
\hline$\Sigma \mathrm{PCBs}$ & $99(100)$ & 2.65 & 1.08 & 0.60 & 1.93 & 2.43 & 3.11 & 7.06 \\
\hline$\Sigma \mathrm{PCDD} / \mathrm{Fs}$ & $99(100)$ & 3.06 & 1.98 & 0.28 & 1.50 & 2.44 & 4.37 & 10.6 \\
\hline$\Sigma \mathrm{PBDD} / \mathrm{Fs}$ & $99(100)$ & 0.91 & 1.07 & 0.15 & 0.35 & 0.60 & 0.93 & 8.42 \\
\hline$\Sigma$ POPs & $99(100)$ & 6.61 & 2.99 & 1.45 & 4.21 & 5.90 & 8.22 & 16.3 \\
\hline
\end{tabular}


Table S6 Comparison of PBDE concentrations in human breast milk measured in different populations (ng/g lipid). Data are shown as median unless specified.

\begin{tabular}{|c|c|c|c|c|c|c|c|c|c|c|c|c|}
\hline \multirow[t]{3}{*}{ POPs } & \multirow[t]{3}{*}{ This study } & \multirow[t]{3}{*}{$\mathrm{US}^{5}$} & \multirow{3}{*}{$\begin{array}{l}\text { Beijing, } \\
\text { China }^{6}\end{array}$} & \multirow{2}{*}{$\begin{array}{l}16 \\
\text { provinces, }\end{array}$} & \multirow{2}{*}{$\begin{array}{l}\text { Taiwan, } \\
\text { China }^{8}\end{array}$} & \multirow{2}{*}{$\begin{array}{l}\text { South } \\
\text { Korea }\end{array}$} & \multirow[t]{3}{*}{ Indonesia ${ }^{10}$} & \multirow[t]{3}{*}{ Japan ${ }^{11}$} & \multirow[t]{3}{*}{ Greece $^{12}$} & \multirow{3}{*}{$\begin{array}{l}\text { Belgium } \\
13\end{array}$} & \multirow[t]{3}{*}{$\mathrm{UK}^{14}$} & \multirow{3}{*}{$\begin{array}{l}\text { Norway } \\
15\end{array}$} \\
\hline & & & & & & & & & & & & \\
\hline & & & & China $^{7}$ & & 9 & & & & & & \\
\hline BDE-17 & $<\mathrm{LOQ}$ & 0.02 & -- & -- & -- & -- & -- & -- & -- & -- & -- & -- \\
\hline BDE-28 & 0.0293 & $1.3-2.0$ & 0.094 & 0.22 & 0.03 & -- & 0.03 & 0.04 & $<0.10$ & $<\mathrm{LOQ}$ & -- & 0.106 \\
\hline BDE-47 & 0.204 & $7.7-31.5$ & 0.066 & 0.25 & 0.22 & 0.54 & 0.30 & 0.68 & 0.48 & 0.16 & 1.92 & 1.031 \\
\hline BDE-66 & 0.0042 & $0.14-0.2$ & -- & -- & -- & -- & -- & -- & -- & -- & -- & -- \\
\hline BDE-85 & 0.0093 & $0.3-0.5$ & -- & -- & -- & -- & -- & -- & -- & -- & -- & -- \\
\hline BDE-99 & 0.0625 & $1.5-6.4$ & 0.016 & 0.08 & 0.06 & 0.29 & 0.15 & 0.41 & 0.27 & 0.06 & 0.88 & 0.271 \\
\hline BDE-100 & 0.0543 & $0.5-5.7$ & 0.016 & 0.06 & 0.08 & 0.12 & 0.11 & 0.38 & 0.19 & 0.06 & -- & 0.257 \\
\hline BDE-153 & 0.377 & $1.1-8.0$ & 0.247 & 0.5 & 0.63 & -- & 0.22 & 0.31 & 0.30 & 0.29 & 1.01 & 0.497 \\
\hline BDE-154 & 0.00825 & $0.2-0.3$ & 0.019 & 0.04 & 0.03 & -- & 0.03 & 0.23 & $<0.10$ & 0.07 & -- & 0.029 \\
\hline
\end{tabular}




\begin{tabular}{|c|c|c|c|c|c|c|c|c|c|c|c|c|}
\hline BDE-183 & 0.0284 & $0.06-0.2$ & 0.027 & 0.32 & 0.05 & -- & 0.09 & -- & $<0.10$ & $<\mathrm{LOQ}$ & 0.05 & -- \\
\hline BDE-196 & 0.0168 & -- & -- & -- & 0.02 & -- & -- & -- & -- & -- & -- & -- \\
\hline BDE-197 & 0.0731 & -- & -- & -- & 0.12 & -- & 0.21 & -- & -- & -- & -- & -- \\
\hline BDE-203 & 0.0167 & -- & -- & -- & 0.02 & -- & -- & -- & -- & -- & -- & -- \\
\hline BDE-206 & 0.0289 & -- & -- & -- & 0.05 & -- & $<0.05$ & -- & -- & -- & -- & -- \\
\hline BDE-207 & 0.0563 & -- & -- & -- & 0.09 & -- & 0.11 & -- & -- & -- & -- & -- \\
\hline BDE-208 & 0.0193 & -- & -- & -- & 0.04 & -- & -- & -- & -- & -- & -- & -- \\
\hline BDE-209 & 0.44 & 1.41 & 2.2 & -- & 0.69 & -- & $<1.3$ & -- & -- & 0.65 & 0.52 & -- \\
\hline
\end{tabular}


Table S7 Comparison of PCB concentrations in human breast milk measured in different populations (ng/g lipid). Data are shown as median unless specified.

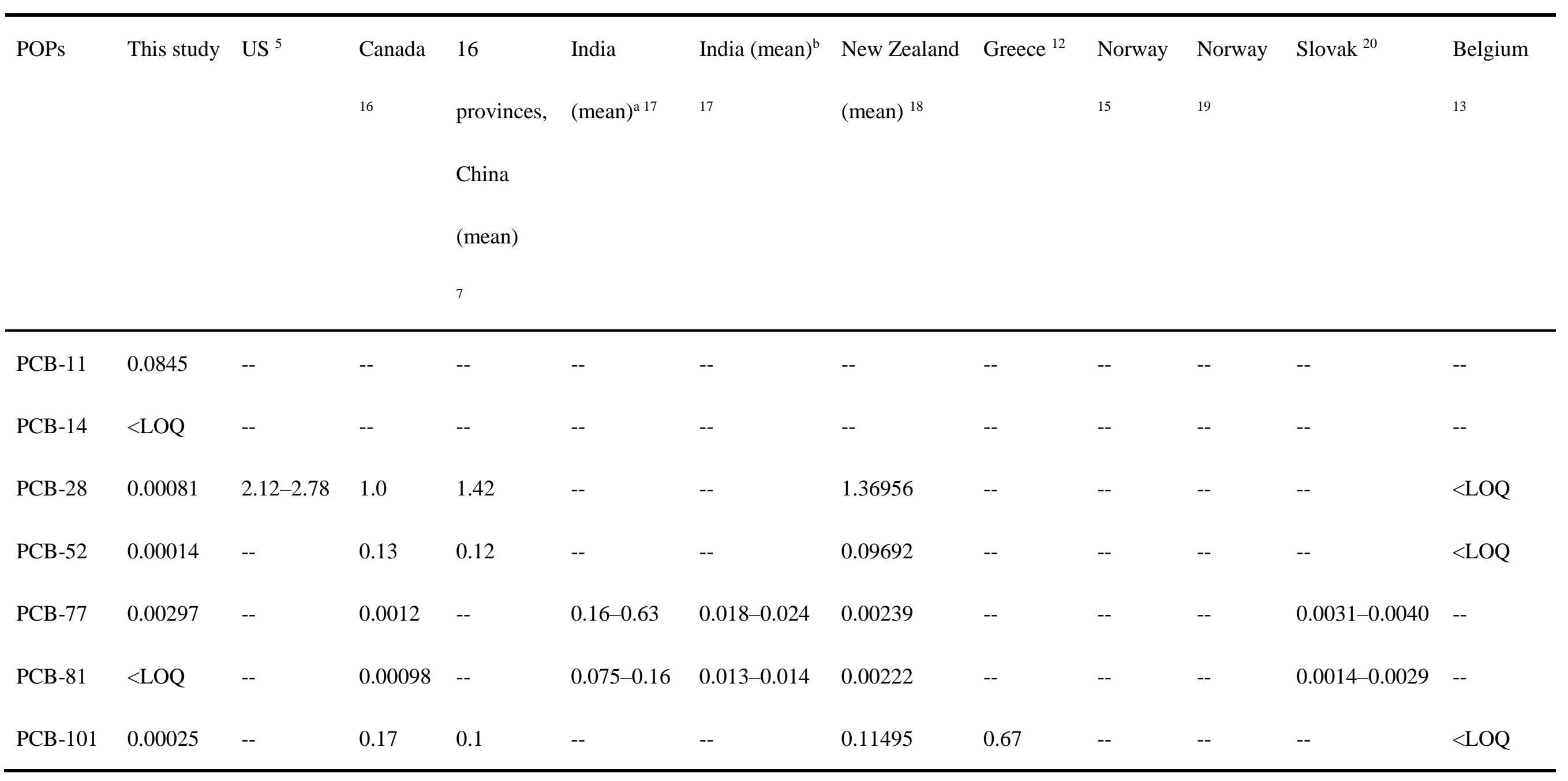




\begin{tabular}{|c|c|c|c|c|c|c|c|c|c|c|c|c|}
\hline PCB-105 & 0.591 & $1.65-1.90$ & 0.67 & -- & $20-23$ & $2.4-2.6$ & 0.34872 & 1.3 & 1.35 & 1.4 & $0.616-0.971$ & 0.7 \\
\hline PCB-114 & 0.224 & -- & 0.18 & -- & $2-2.8$ & $0.34-0.35$ & 0.06929 & -- & 0.335 & 0.35 & $0.123-0.185$ & -- \\
\hline PCB-118 & 3.619 & $6.10-7.97$ & 2.9 & -- & $57-66$ & $6.8-8.3$ & 1.27967 & 4.6 & 5.945 & 6.82 & $4.213-5.078$ & 3.7 \\
\hline PCB-123 & 0.0376 & -- & 0.05 & -- & $1.1-1.3$ & $0.14-0.20$ & 0.02190 & -- & -- & -- & $0.039-0.0447$ & -- \\
\hline PCB-126 & 0.0181 & 0.012 & 0.0097 & -- & $0.16-0.21$ & $0.039-0.049$ & 0.01009 & -- & -- & -- & $0.0201-0.0325$ & -- \\
\hline PCB-138 & 0.014 & $0.04-0.18^{\mathrm{a}}$ & 5.1 & 2.22 & -- & -- & 4.82487 & 13 & 19.412 & 20.3 & -- & 13.5 \\
\hline PCB-153 & 0.0233 & $17-22.8$ & 7.9 & 1.83 & -- & -- & 5.55128 & 24 & 30.9 & 34.7 & -- & 16.8 \\
\hline PCB-156 & 2.128 & $2.04-5.39$ & 0.93 & -- & $8.2-12$ & $1.8-1.9$ & 0.68449 & 2.1 & 3.21 & 3.34 & $4.381-5.632$ & -- \\
\hline PCB-157 & 0.344 & -- & 0.21 & -- & $1.9-2.7$ & $0.44-0.46$ & 0.13521 & -- & 0.67 & 0.61 & $0.4-0.495$ & -- \\
\hline PCB-167 & 0.662 & -- & 0.23 & -- & $3.2-4.0$ & $0.55-0.69$ & 0.18064 & -- & 0.78 & 0.84 & $1.132-1.403$ & -- \\
\hline PCB-169 & 0.0109 & 0.0074 & 0.0045 & -- & $0.014-$ & $0.011-0.012$ & 0.00666 & -- & -- & -- & $0.0098-0.0216$ & -- \\
\hline & & & & & 0.024 & & & & & & & \\
\hline PCB-180 & 0.0132 & $7.78-10.6$ & 4.0 & 0.9 & -- & -- & 2.91207 & 13 & 15.169 & 17.7 & -- & 8.8 \\
\hline PCB-189 & 0.219 & -- & 0.069 & -- & $0.45-0.62$ & $0.17-0.21$ & 0.05285 & -- & 0.23 & 0.25 & $0.478-0.748$ & -- \\
\hline
\end{tabular}


adumping site

${ }^{\mathrm{b}}$ reference site 
Table S8 Comparison of dioxin concentrations in human breast milk measured in different populations (pg/g lipid). Data are shown as median unless specified.

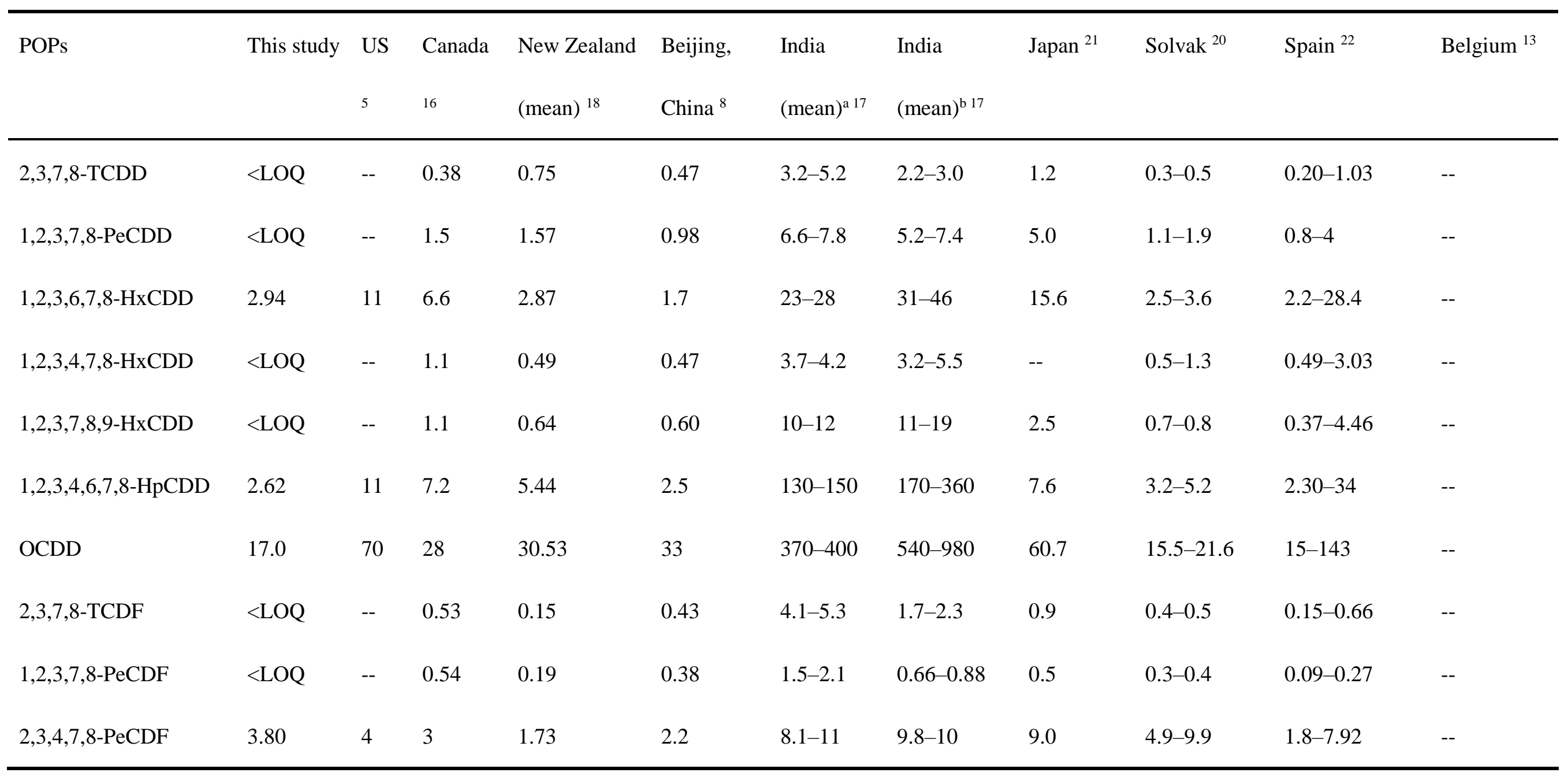




\begin{tabular}{|c|c|c|c|c|c|c|c|c|c|c|c|}
\hline $1,2,3,4,7,8-\mathrm{HxCDF}$ & 1.01 & 1.9 & 5.2 & 0.64 & 0.77 & $4-4.3$ & $4.2-4.8$ & 3.2 & $2.3-3.8$ & $0.66-3.08$ & -- \\
\hline $1,2,3,6,7,8-\mathrm{HxCDF}$ & 1.03 & 1.6 & 2.2 & 0.58 & 0.73 & $4.7-5.1$ & $4.8-6.6$ & 3.5 & $2-3.3$ & $0.63-2.51$ & -- \\
\hline $1,2,3,7,8,9-\mathrm{HxCDF}$ & $<\mathrm{LOQ}$ & -- & $<\mathrm{LOD}$ & 0.36 & 0.12 & 0.039 & $<0.6$ & -- & $0.05-0.1$ & $0.05-0.99$ & -- \\
\hline $2,3,4,6,7,8-\mathrm{HxCDF}$ & $<\mathrm{LOQ}$ & -- & 0.82 & 0.38 & 0.44 & $1.6-1.8$ & $2.3-2.4$ & 2.3 & $0.6-1.5$ & $0.14-0.31$ & -- \\
\hline $1,2,3,4,6,7,8-\mathrm{HpCDF}$ & 0.84 & 2 & 5.4 & 1.72 & 0.76 & $13-14$ & $18-30$ & 1.6 & $0.7-1.3$ & $0.05-2.17$ & -- \\
\hline 1,2,3,4,7,8,9-HpCDF & $<\mathrm{LOQ}$ & -- & 1.2 & 0.43 & 0.16 & 0.17 & $0.52-0.63$ & -- & 0.1 & $0.07-0.17$ & -- \\
\hline OCDF & $<\mathrm{LOQ}$ & 0.17 & 14 & 0.54 & 0.26 & 2 & $1-1.1$ & -- & $0.1-0.2$ & $0.31-0.43$ & -- \\
\hline 2,3,7,8-TBDD & $<\mathrm{LOQ}$ & -- & -- & -- & -- & -- & -- & -- & -- & -- & 0.05 \\
\hline 1,2,3,7,8-PeBDD & $<\mathrm{LOQ}$ & -- & -- & -- & -- & -- & -- & -- & -- & -- & $<0.07$ \\
\hline 1,2,3,4,7,8-HxBDD & $<\mathrm{LOQ}$ & -- & -- & -- & -- & -- & -- & -- & -- & -- & $<0.07$ \\
\hline 1,2,3,6,7,8-HxBDD & $<\mathrm{LOQ}$ & -- & -- & -- & -- & -- & -- & -- & -- & -- & -- \\
\hline 1,2,3,7,8,9-HxBDD & $<\mathrm{LOQ}$ & -- & -- & -- & -- & -- & -- & -- & -- & -- & $<0.04$ \\
\hline 1,2,3,4,6,7,8-HpBDD & $<\mathrm{LOQ}$ & -- & -- & -- & -- & -- & -- & -- & -- & -- & -- \\
\hline OBDD & $<\mathrm{LOQ}$ & -- & -- & -- & -- & -- & -- & -- & -- & -- & \\
\hline
\end{tabular}




\begin{tabular}{|c|c|c|c|c|c|c|c|c|c|c|c|}
\hline $2,3,7,8-\mathrm{TBDF}$ & $<\mathrm{LOQ}$ & -- & -- & -- & -- & -- & -- & -- & -- & -- & 0.7 \\
\hline 1,2,3,7,8-PeBDF & $<\mathrm{LOQ}$ & -- & -- & -- & -- & -- & -- & -- & -- & -- & 0.2 \\
\hline 2,3,4,7,8-PeBDF & $<\mathrm{LOQ}$ & -- & -- & -- & -- & -- & -- & -- & -- & -- & 0.4 \\
\hline $1,2,3,4,7,8-\mathrm{HxBDF}$ & $<\mathrm{LOQ}$ & -- & -- & -- & -- & -- & -- & -- & -- & -- & $<0.3$ \\
\hline $1,2,3,4,6,7,8-\mathrm{HpBDF}$ & $<\mathrm{LOQ}$ & -- & -- & -- & -- & -- & -- & -- & -- & -- & -- \\
\hline OBDF & $<\mathrm{LOQ}$ & -- & -- & -- & -- & -- & -- & -- & -- & -- & -- \\
\hline
\end{tabular}

adumping site

${ }^{\mathrm{b}}$ reference site 
Table S9 Correlations between demographic characteristics and THs in human breast milk.

Comparisons were performed using Welch t-test or analysis of variance (ANOVA).

\begin{tabular}{|c|c|c|c|c|}
\hline & $\mathrm{N}(\%)$ & $\mathrm{TT}_{4}(\mathrm{ng} / \mathrm{mL})$ & $\mathrm{TT}_{3}(\mathrm{ng} / \mathrm{mL})$ & $\mathrm{TrT}_{3}(\mathrm{ng} / \mathrm{mL})$ \\
\hline \multicolumn{5}{|c|}{ Maternal age (y) } \\
\hline$\leq 29$ & $15(15.2)$ & $0.75^{* *}$ & $0.15^{* *}$ & $0.03 * *$ \\
\hline 30-39 & $75(75.8)$ & 0.55 & 0.13 & 0.02 \\
\hline$\geq 40$ & $9(9.1)$ & 0.49 & 0.11 & 0.02 \\
\hline \multicolumn{5}{|c|}{ Maternal BMI (kg/m²) } \\
\hline$<24.9$ & $66(66.7)$ & 0.58 & 0.13 & 0.02 \\
\hline $25-29.9$ & $19(19.2)$ & 0.57 & 0.14 & 0.02 \\
\hline$\geq 30$ & $11(11.1)$ & 0.58 & 0.13 & 0.02 \\
\hline Missing & $3(3.0)$ & 0.47 & 0.12 & 0.03 \\
\hline \multicolumn{5}{|l|}{ Education } \\
\hline$<12$ years & $37(37.4)$ & $0.62 \#$ & 0.13 & 0.02 \\
\hline$\geq 12$ years & $62(62.6)$ & 0.55 & 0.13 & 0.02 \\
\hline \multicolumn{5}{|l|}{ Parity } \\
\hline 1 & $45(45.5)$ & 0.60 & 0.13 & 0.02 \\
\hline 2 & $46(46.5)$ & 0.56 & 0.13 & 0.02 \\
\hline$\geq 3$ & $8(8.1)$ & 0.55 & 0.14 & 0.02 \\
\hline \multicolumn{5}{|c|}{ Time of breastfeeding (month) } \\
\hline Never & $10(10.1)$ & 0.63 & 0.12 & 0.02 \\
\hline $1-3$ & $20(20.2)$ & 0.59 & 0.12 & 0.02 \\
\hline $3-5$ & $40(40.4)$ & 0.55 & 0.13 & 0.02 \\
\hline$\geq 5$ & $24(24.2)$ & 0.58 & 0.13 & 0.02 \\
\hline Missing & $5(5.1)$ & 0.59 & 0.15 & 0.02 \\
\hline Ethnicity & & & & \\
\hline
\end{tabular}




\begin{tabular}{|c|c|c|c|c|}
\hline German & $89(89.9)$ & $0.55 * *$ & $0.13^{*}$ & 0.02 \\
\hline Non-German & $10(10.1)$ & 0.80 & 0.15 & 0.03 \\
\hline \multicolumn{5}{|c|}{ Maternal smoking } \\
\hline No & $95(95.0)$ & 0.58 & 0.13 & 0.02 \\
\hline Yes & $4(4.0)$ & 0.48 & 0.12 & 0.02 \\
\hline \multicolumn{5}{|l|}{ Vegetarian } \\
\hline Yes & $18(18.2)$ & 0.55 & 0.13 & 0.02 \\
\hline No & $81(81.8)$ & 0.58 & 0.13 & 0.02 \\
\hline \multicolumn{5}{|c|}{ Infant age at sampling (d) } \\
\hline$<60$ & $19(19.2)$ & $0.68 * *$ & 0.13 & $0.03^{* *}$ \\
\hline $60-120$ & $37(37.4)$ & 0.54 & 0.13 & 0.02 \\
\hline $120-180$ & $28(28.3)$ & 0.50 & 0.13 & 0.02 \\
\hline$\geq 180$ & $14(14.1)$ & 0.64 & 0.13 & 0.02 \\
\hline \multicolumn{5}{|l|}{ Infant gender } \\
\hline Female & $47(47.5)$ & 0.61 & 0.13 & 0.02 \\
\hline Male & $48(48.5)$ & 0.54 & 0.13 & 0.02 \\
\hline Missing & $4(4.0)$ & 0.55 & 0.14 & 0.02 \\
\hline
\end{tabular}

$* p<0.05 ; * * p<0.01 . \# p<0.10$ 
Table S10 Correlations between demographic characteristics and PBDEs in breast milk. Comparisons were performed using Welch t-test or analysis of variance (ANOVA). PBDE concentrations were expressed as $\mathrm{pg} / \mathrm{mL}$ milk and $\mathrm{pg} / \mathrm{g}$ lw (in the parentheses).

\begin{tabular}{|c|c|c|c|c|c|c|c|c|c|c|c|c|c|c|c|}
\hline & $\mathrm{N}(\%)$ & BDE- & BD- & BDE- & BDE- & BDE- & BDE- & BDE- & BDE- & BDE- & BDE- & BDE- & BDE- & BDE- & BDE- \\
\hline & & 28 & E47 & 99 & 100 & 153 & 154 & 183 & 196 & 197 & 203 & 206 & 207 & 208 & 209 \\
\hline \multicolumn{16}{|c|}{ Maternal age (y) } \\
\hline \multirow[t]{2}{*}{$\leq 29$} & 15 & 0.87 & 8.43 & 2.33 & 2.31 & 18.8 & 0.24 & 1.08 & 0.76 & 2.89 & 0.65 & 8.01 & 7.43 & 3.72 & 227 \\
\hline & (15.2) & (29.6) & $(275)$ & (78.4) & $(72.4)$ & (528) & $(7.91)$ & $(32.2)$ & $(23.3)$ & (89.7) & (19.4) & (256) & (236) & (119) & (7293) \\
\hline \multirow[t]{2}{*}{$30-39$} & 75 & 0.91 & 9.73 & 2.56 & 2.24 & 13.3 & 0.26 & 0.99 & 0.55 & 2.39 & 0.58 & 3.74 & 3.60 & 1.60 & 101 \\
\hline & $(75.8)$ & (30.8) & (324) & $(87.6)$ & $(74.8)$ & $(445)$ & $(8.81)$ & $(33.7)$ & $(20.2)$ & $(82.6)$ & (21.9) & (149) & (138) & (61.5) & $(4120)$ \\
\hline \multirow[t]{2}{*}{$\geq 40$} & $9(9.1)$ & 1.00 & 7.60 & 2.50 & 1.82 & 17.0 & 0.35 & 1.05 & 0.62 & 2.75 & 0.64 & 0.80 & 2.05 & 0.84 & 15.7 \\
\hline & & $(29.5)$ & (221) & $(75.4)$ & $(52.4)$ & (468) & $(9.48)$ & $(29.0)$ & (17.7) & (77.7) & (18.5) & $(22.5)$ & (59.8) & (24.7) & (430) \\
\hline \multicolumn{16}{|c|}{ Maternal BMI $\left(\mathrm{kg} / \mathrm{m}^{2}\right)$} \\
\hline \multirow[t]{2}{*}{$<24.9$} & 66 & 0.92 & 9.77 & 2.52 & 2.19 & 15.5 & 0.25 & $0.86 \#$ & 0.54 & 2.45 & 0.59 & 2.83 & 3.09 & 1.36 & 75.0 \\
\hline & $(66.7)$ & $(30.9)$ & (312) & $(82.7)$ & $(70.4)$ & (494\#) & $(8.24)$ & $(28.4 \#)$ & $(18.9)$ & $(81.6)$ & $(21.6)$ & $(113)$ & $(117)$ & $(51.6)$ & $(3080)$ \\
\hline
\end{tabular}




\begin{tabular}{|c|c|c|c|c|c|c|c|c|c|c|c|c|c|c|c|}
\hline \multirow[t]{2}{*}{$25-29.9$} & 19 & 0.90 & 9.16 & 2.74 & 2.32 & 11.6 & 0.31 & 1.14 & 0.77 & 2.62 & 0.67 & 9.30 & 7.80 & 3.81 & 269 \\
\hline & (19.2) & $(32.7)$ & $(358)$ & (109) & $(86.8)$ & (399) & $(10.9)$ & $(40.3)$ & $(28.6)$ & $(94.4)$ & $(24.4)$ & (339) & $(278)$ & (135) & (9785) \\
\hline \multirow[t]{2}{*}{$\geq 30$} & 11 & 0.81 & 8.46 & 2.44 & 2.45 & 12.4 & 0.26 & 1.22 & 0.47 & 2.42 & 0.47 & 1.53 & 2.42 & 1.02 & 33.1 \\
\hline & $(11.1)$ & $(23.7)$ & $(240)$ & $(70.4)$ & $(70.6)$ & $(350)$ & $(8.05)$ & $(36.8)$ & $(14.3)$ & $(71.1)$ & $(13.2)$ & $(44.2)$ & $(69.7)$ & (29.9) & (1007) \\
\hline \multirow[t]{2}{*}{ Missing } & $3(3.0)$ & 1.16 & 4.29 & 1.43 & 1.15 & 17.1 & 0.29 & 2.62 & 0.84 & 3.21 & 0.75 & 9.15 & 7.09 & 3.34 & 239 \\
\hline & & (33.9) & (125) & $(41.8)$ & $(33.4)$ & $(497)$ & $(8.40)$ & (76.2) & $(25.0)$ & $(92.5)$ & $(22.0)$ & (279) & $(215)$ & (101) & (7316) \\
\hline \multicolumn{16}{|c|}{ Education (y) } \\
\hline \multirow[t]{2}{*}{$<12$} & 37 & $0.79 \#$ & 8.30 & 2.31 & 2.08 & 13.8 & 0.26 & 1.02 & 0.60 & 2.52 & 0.54 & $4.39 *$ & 4.45 & 2.11 & $121 \#$ \\
\hline & $(37.4)$ & $(26.5 \#)$ & (269) & $(77.5)$ & $(68.5)$ & $(436)$ & $(8.41)$ & $(34.8)$ & (19.8) & $(84.5)$ & $(18.5)$ & $\left(144^{*}\right)$ & $(147)$ & $(69.3)$ & (3946\#) \\
\hline \multirow[t]{2}{*}{$\geq 12$} & 62 & 0.98 & 9.96 & 2.65 & 2.29 & 14.9 & 0.27 & 1.00 & 0.58 & 2.49 & 0.62 & 3.95 & 3.80 & 1.69 & 108 \\
\hline & $(62.6)$ & (32.9) & (330) & (89.6) & (74.8) & $(474)$ & $(8.93)$ & $(32.0)$ & (20.8) & $(82.5)$ & $(22.8)$ & (159) & $(145)$ & $(65.3)$ & (4456) \\
\hline \multicolumn{16}{|l|}{ Parity } \\
\hline \multirow[t]{2}{*}{1} & 45 & 0.97 & 8.85 & $2.26 *$ & 2.06 & 15.9 & 0.24 & 1.01 & 0.58 & 2.49 & 0.57 & 5.00 & 4.63 & 2.16 & 137 \\
\hline & $(45.5)$ & $(34.2)$ & (293) & (77.8\#) & $(68.7)$ & $(506)$ & $(8.41)$ & $(32.8)$ & (19.5) & $(81.6)$ & (19.0) & (164) & (154) & (71.4) & (4490) \\
\hline
\end{tabular}




\begin{tabular}{|c|c|c|c|c|c|c|c|c|c|c|c|c|c|c|c|}
\hline 2 & 46 & 0.85 & 9.85 & 2.70 & 2.29 & 13.2 & 0.28 & 1.02 & 0.59 & 2.50 & 0.62 & 3.68 & 3.71 & 1.68 & 102 \\
\hline \multirow{3}{*}{$\geq 3$} & $(46.5)$ & $(27.0)$ & (324) & (90.9) & $(74.4)$ & $(420)$ & $(9.06)$ & (33.6) & (21.6) & $(84.8)$ & $(24.1)$ & (163) & $(150)$ & $(69.0)$ & $(4651)$ \\
\hline & \multirow[t]{2}{*}{$8(8.1)$} & 0.90 & 9.15 & 2.99 & 2.62 & 13.9 & 0.27 & 0.95 & 0.62 & 2.56 & 0.56 & 1.67 & 2.68 & 1.09 & 33.7 \\
\hline & & $(30.3)$ & (286) & $(92.4)$ & (81.9) & $(430)$ & $(8.74)$ & $(31.2)$ & (18.9) & $(83.0)$ & $(17.0)$ & $(38.6)$ & (71.9) & $(28.2)$ & (779) \\
\hline \multicolumn{16}{|c|}{ Time of breastfeeding (month) } \\
\hline \multirow[t]{2}{*}{0} & 10 & 1.00 & 7.17 & 2.17 & 2.31 & 12.6 & 0.30 & 1.29 & 0.52 & 2.46 & 0.43 & $0.73 *$ & $1.65^{*}$ & $0.57 *$ & $13.2 *$ \\
\hline & $(10.1)$ & $(35.4)$ & $(254)$ & $(76.3)$ & $(80.1)$ & $(432)$ & $(10.3)$ & $(43.4)$ & $(17.6 \#)$ & $(84.7)$ & $(15.1 \#)$ & $(23.6 *)$ & $(55.8 * *)$ & $(19.2 *)$ & $(410 *)$ \\
\hline \multirow[t]{2}{*}{$1-3$} & 20 & 0.87 & 8.22 & 2.30 & 2.19 & 17.6 & 0.25 & 1.05 & 0.88 & 3.03 & 0.83 & 12.5 & 10.3 & 5.10 & 359 \\
\hline & $(20.2)$ & $(29.8)$ & $(288)$ & $(83.8)$ & $(71.4)$ & $(519)$ & $(8.65)$ & $(34.0)$ & $(29.2)$ & $(99.6)$ & $(27.2)$ & $(410)$ & $(337)$ & (166) & (11811) \\
\hline \multirow[t]{2}{*}{$3-5$} & 40 & 0.93 & 10.6 & 2.69 & 2.41 & 14.2 & 0.27 & 1.02 & 0.53 & 2.37 & 0.57 & 2.27 & 2.62 & 1.12 & 60.9 \\
\hline & $(40.4)$ & $(32.4)$ & (354) & (92.8) & (81.1) & (446) & $(9.17)$ & $(34.2)$ & (20.8) & $(81.9)$ & $(23.8)$ & (126) & (123) & $(55.4)$ & $(3500)$ \\
\hline \multirow[t]{2}{*}{$\geq 5$} & 24 & 0.85 & 9.58 & 2.68 & 1.99 & 12.0 & 0.25 & 0.86 & 0.49 & 2.29 & 0.50 & 2.38 & 2.76 & 1.17 & 56.1 \\
\hline & $(24.2)$ & $(24.7)$ & $(280)$ & $(80.3)$ & $(58.7)$ & $(401)$ & $(7.55)$ & $(26.5)$ & (14.7) & (70.6) & (15.1) & (68.9) & $(80.8)$ & $(33.2)$ & (1671) \\
\hline
\end{tabular}




\begin{tabular}{|c|c|c|c|c|c|c|c|c|c|c|c|c|c|c|c|}
\hline \multirow[t]{2}{*}{ Missing } & $5(5.1)$ & 1.07 & 6.79 & 1.96 & 1.56 & 20.4 & 0.23 & 0.88 & 0.45 & 2.55 & 0.56 & 0.56 & 1.55 & 0.54 & 10.3 \\
\hline & & $(37.1)$ & (239) & $(68.3)$ & $(57.2)$ & $(682)$ & $(8.14)$ & $(30.3)$ & $(15.5)$ & $(85.7)$ & $(18.1)$ & $(19.3)$ & $(50.9)$ & $(17.5)$ & $(367)$ \\
\hline \multicolumn{16}{|l|}{ Ethnicity } \\
\hline \multirow[t]{2}{*}{ German } & 89 & 0.90 & 9.48 & 2.56 & 2.25 & 14.8 & 0.26 & 1.00 & 0.58 & 2.45 & 0.60 & 4.04 & 4.07 & 1.86 & 110 \\
\hline & $(89.9)$ & $(31.0)$ & (316) & $(87.9 *)$ & (74.8\#) & $(476 * *)$ & $(8.92)$ & $(33.5)$ & $(20.8)$ & $(83.3)$ & $(21.9 *)$ & (155) & (149) & $(68.6)$ & $(4300)$ \\
\hline \multirow[t]{2}{*}{ Non-German } & 10 & 0.99 & 8.08 & 2.14 & 1.88 & 11.4 & 0.27 & 1.04 & 0.61 & 2.98 & 0.51 & 4.76 & 3.78 & 1.72 & 135 \\
\hline & $(10.1)$ & $(26.7)$ & $(225)$ & $(60.1)$ & $(51.0)$ & (316) & $(7.09)$ & $(28.6)$ & $(17.1)$ & $(82.2)$ & $(14.7)$ & (138) & (114) & $(50.5)$ & (3956) \\
\hline \multicolumn{16}{|c|}{ Maternal smoking } \\
\hline \multirow[t]{2}{*}{ No } & 95 & 0.90 & 9.39 & 2.53 & 2.20 & 14.6 & 0.26 & 1.01 & 0.59 & 2.50 & 0.59 & $4.11 \#$ & 4.03 & 1.85 & 114 \\
\hline & $(95.0)$ & (30.7) & (313) & (86.6) & (73.3) & $(468)$ & (8.86) & (33.6) & (20.7) & (84.4) & (21.4) & (157) & (148) & (67.9) & (4377) \\
\hline \multirow[t]{2}{*}{ Yes } & $4(4.0)$ & 1.21 & 8.19 & 2.28 & 2.43 & 12.7 & 0.27 & 0.88 & 0.60 & 2.52 & 0.76 & 4.18 & 4.31 & 1.96 & 80.0 \\
\hline & & (26.0) & (172) & (48.4) & $(50.6)$ & (277) & $(5.86)$ & (18.9) & (12.7) & $(56.0)$ & (16.0) & $(84.6)$ & (88.7) & $(40.2)$ & (1612) \\
\hline Vegetarian & & & & & & & & & & & & & & & \\
\hline
\end{tabular}




\begin{tabular}{|c|c|c|c|c|c|c|c|c|c|c|c|c|c|c|c|}
\hline Yes & 18 & 1.02 & 8.37 & 2.12 & 2.33 & 19.9\# & 0.28 & 1.28 & $0.77 *$ & 3.06 & $0.93 *$ & $7.11 *$ & $6.16^{*}$ & $2.91 *$ & 202\# \\
\hline \multirow{3}{*}{ No } & $(18.2)$ & $(29.8)$ & $(240)$ & $(65.2)$ & $(66.3)$ & $(545)$ & $(8.15)$ & $(37.4)$ & $(27.5 \#)$ & $(91.5)$ & $(35.8 *)$ & $\left(294^{*}\right)$ & $(248 \#)$ & $(117 \#)$ & $(8630 \#)$ \\
\hline & 81 & 0.89 & 9.55 & 2.61 & 2.19 & 13.3 & 0.26 & 0.95 & 0.55 & 2.38 & 0.52 & 3.45 & 3.57 & 1.61 & 92.5 \\
\hline & $(81.8)$ & $(30.7)$ & $(322)$ & $(89.5)$ & $(73.8)$ & $(441)$ & $(8.87)$ & $(32.1)$ & $(18.9)$ & $(81.4)$ & $(18.0)$ & $(122)$ & $(123)$ & $(55.6)$ & $(3295)$ \\
\hline \multicolumn{16}{|c|}{ Infant age at sampling (d) } \\
\hline \multirow[t]{2}{*}{$<60$} & 19 & 0.94 & 9.21 & 2.42 & 2.08 & 15.2 & 0.24 & 0.88 & 0.86 & 2.72 & 0.76 & 13.6 & $10.9 *$ & $5.51 \#$ & 390\# \\
\hline & (19.2) & (30.4) & (275) & (73.3) & $(64.9)$ & (466) & (7.64) & (27.1) & $(27.1)$ & (82.3) & (23.3) & (426) & (341) & (172) & (12276) \\
\hline \multirow[t]{2}{*}{$60-120$} & 37 & 0.81 & 7.27 & 2.07 & 2.14 & 14.4 & 0.26 & 1.16 & 0.54 & 2.41 & 0.53 & 1.74 & 2.15 & 0.85 & 42.1 \\
\hline & (37.4) & (29.7) & $(270)$ & $(78.8)$ & (75.6) & (477) & $(9.54)$ & $(40.0)$ & $(20.5)$ & $(85.6)$ & (19.7) & $(83.5)$ & (89.4) & $(36.9)$ & (2112) \\
\hline \multirow[t]{2}{*}{$120-180$} & 28 & 0.93 & 8.90 & 2.57 & 2.02 & 13.7 & 0.27 & 0.91 & 0.56 & 2.49 & 0.66 & 2.43 & 2.89 & 1.23 & 64.9 \\
\hline & (28.3) & (31.3) & (309) & (91.7) & $(67.4)$ & $(481)$ & $(8.70)$ & $(30.8)$ & (20.7) & $(87.0)$ & $(26.5)$ & (128) & (133) & $(58.5)$ & $(3651)$ \\
\hline \multirow[t]{2}{*}{$\geq 180$} & 14 & 1.03 & 15.9 & 3.89 & 3.09 & 15.9 & 0.30 & 1.00 & 0.41 & 2.51 & 0.44 & 1.19 & 2.26 & 0.86 & 24.6 \\
\hline & (14.1) & $(29.2)$ & $(447)$ & (110) & (88.4) & (379) & $(8.41)$ & $(28.2)$ & (11.6) & (73.1) & (12.8) & $(30.1)$ & $(61.1)$ & $(23.2)$ & (594) \\
\hline Infant ger & & & & & & & & & & & & & & & \\
\hline
\end{tabular}




\begin{tabular}{|c|c|c|c|c|c|c|c|c|c|c|c|c|c|c|c|}
\hline \multirow[t]{2}{*}{ Female } & 47 & 0.93 & 8.38 & 2.39 & 2.42 & 14.5 & 0.26 & 1.07 & 0.62 & 2.63 & 0.60 & $3.96 \#$ & 3.66 & $1.62 \#$ & 104 \\
\hline & $(47.5)$ & (29.6) & (259) & (75.9) & (73.8) & (437) & $(8.25)$ & $(33.8)$ & $(20.5)$ & $(81.8)$ & (19.3) & (138) & (123) & $(54.7)$ & $(3685)$ \\
\hline \multirow[t]{2}{*}{ Male } & 48 & 0.91 & 10.6 & 2.74 & 2.09 & 14.5 & 0.27 & 0.97 & 0.58 & 2.38 & 0.61 & 4.58 & 4.64 & 2.19 & 130 \\
\hline & $(48.5)$ & $(31.8)$ & $(360)$ & (96.2) & (72.4) & $(481)$ & $(9.36)$ & (32.9) & (21.2) & $(84.7)$ & $(23.8)$ & (180) & $(176)$ & $(83.1)$ & (5168) \\
\hline \multirow[t]{2}{*}{ Missing } & $4(4.0)$ & 0.71 & 5.69 & 1.51 & 1.30 & 14.6 & 0.18 & 0.68 & 0.28 & 2.29 & 0.35 & 0.41 & 1.35 & 0.40 & 6.77 \\
\hline & & $(26.0)$ & $(232)$ & $(59.8)$ & $(56.5)$ & (483) & (6.98) & $(25.5)$ & $(10.2)$ & $(81.9)$ & (11.9) & (14.9) & (47.3) & (14.3) & (237) \\
\hline
\end{tabular}

${ }^{*} p<0.05 ;{ }^{* *} p<0.01 . \# p<0.10$ 
Table S11 Correlations between demographic characteristics and PCBs in breast milk. Comparisons were performed using Welch t-test or analysis of variance (ANOVA). PCB concentrations were expressed as $\mathrm{pg} / \mathrm{mL}$ milk and $\mathrm{pg} / \mathrm{g}$ lw (in the parentheses).

\begin{tabular}{|c|c|c|c|c|c|c|c|c|c|c|c|c|c|c|c|c|c|c|}
\hline & $\mathrm{N}(\%)$ & PCB- & PCB- & PCB- & PCB- & PCB- & PCB- & PCB- & PCB- & PCB- & PCB- & PCB- & PCB- & PCB- & PCB- & PCB- & PCB- & PCB- \\
\hline & & 77 & 126 & 169 & 105 & 114 & 118 & 123 & 156 & 157 & 167 & 189 & 28 & 52 & 101 & 138 & 153 & 180 \\
\hline \multicolumn{19}{|c|}{ Maternal age (y) } \\
\hline \multirow[t]{2}{*}{$\leq 29$} & 15 & 0.10 & 0.59 & 0.34 & 21.8 & 8.29 & 121 & 1.31 & 87.5 & 13.2 & 20.8 & 7.10\# & 0.04 & 0.01 & 0.01 & 0.51 & 0.87 & $0.46 \#$ \\
\hline & (15.2) & $(2.93)$ & (18.5) & (10.6) & $(671)$ & (251) & $(3750)$ & $(40.5)$ & (2634) & (396) & $(637)$ & $\left(213^{*}\right)$ & $(1.10)$ & $(0.15)$ & $(0.28)$ & (15.8) & $(26.5)$ & (13.9\#) \\
\hline \multirow[t]{2}{*}{$30-39$} & 75 & 0.09 & 0.60 & 0.37 & 19.6 & 7.32 & 116 & 1.27 & 77.5 & 11.4 & 19.9 & 7.43 & 0.03 & 0.01 & 0.01 & 0.43 & 0.75 & 0.46 \\
\hline & $(75.8)$ & (3.34) & (20.3) & (12.6) & $(659)$ & (245) & (3943) & (42.9) & (2603) & (384) & $(680)$ & $(250)$ & $(1.00)$ & $(0.19)$ & $(0.41)$ & (14.7) & $(25.5)$ & (15.5) \\
\hline \multirow[t]{2}{*}{$\geq 40$} & $9(9.1)$ & 0.07 & 0.66 & 0.48 & 22.4 & 9.82 & 140 & 1.20 & 111 & 15.7 & 27.6 & 10.9 & 0.02 & 0.00 & 0.01 & 0.62 & 1.12 & 0.68 \\
\hline & & $(2.32)$ & (20.6) & (14.6) & (719) & (298) & (4390) & (37.4) & (3272) & (467) & $(844)$ & (324) & $(0.74)$ & $(0.14)$ & $(0.26)$ & (18.1) & (33.0) & (20.4) \\
\hline
\end{tabular}




\begin{tabular}{|c|c|c|c|c|c|c|c|c|c|c|c|c|c|c|c|c|c|c|}
\hline \multirow[t]{2}{*}{$<24.9$} & 66 & 0.09 & 0.60 & 0.39 & 20.1 & 8.01 & 119 & 1.29 & $86.5 \#$ & 12.8 & 21.4 & $8.25 *$ & 0.03 & 0.01 & 0.01 & 0.46 & 0.82 & $0.52 *$ \\
\hline & $(66.7)$ & $(3.28)$ & $(20.2)$ & $(13.3 * *)$ & $(674)$ & $\left(266^{*}\right)$ & (4003) & $(43.7 \#)$ & $(2897 *$ & $(426 * *)$ & $(726 *)$ & $(277 * *)$ & $(0.97)$ & $(0.17)$ & $(0.33)$ & $(15.6)$ & $(27.8 *)$ & $(17.3 * *)$ \\
\hline & & & & & & & & & \multicolumn{10}{|l|}{$*)$} \\
\hline \multirow[t]{2}{*}{$25-29.9$} & 19 & 0.09 & 0.58 & 0.31 & 19.7 & 6.32 & 114 & 1.14 & 64.6 & 9.59 & 18.0 & 5.75 & 0.03 & 0.01 & 0.01 & 0.42 & 0.67 & 0.37 \\
\hline & $(19.2)$ & $(3.02)$ & (20.6) & $(10.5)$ & $(680)$ & (209) & (3928) & $(39.3)$ & (2076) & (311) & $(611)$ & (185) & $(0.97)$ & $(0.22)$ & $(0.29)$ & (14.0) & $(22.4)$ & (11.9) \\
\hline \multirow[t]{2}{*}{$\geq 30$} & 11 & 0.10 & 0.61 & 0.28 & 18.1 & 6.25 & 115 & 1.15 & 65.3 & 9.25 & 17.7 & 5.91 & 0.04 & 0.01 & 0.03 & 0.43 & 0.70 & 0.36 \\
\hline & $(11.1)$ & $(3.00)$ & (18.0) & $(8.21)$ & $(523)$ & (180) & (3353) & $(33.4)$ & $(1840)$ & $(266)$ & (509) & $(162)$ & (1.02) & $(0.23)$ & $(0.79)$ & $(12.2)$ & $(20.0)$ & $(10.2)$ \\
\hline \multirow[t]{2}{*}{ Missing } & $3(3.0)$ & 0.09 & 0.74 & 0.76 & 32.5 & 14.8 & 182 & 1.88 & 154 & 22.7 & 34.5 & 14.1 & 0.05 & 0.01 & 0.01 & 0.84 & 1.44 & 0.86 \\
\hline & & $(2.66)$ & $(21.5)$ & $(22.0)$ & (939) & (428) & $(5265)$ & $(54.3)$ & (4431) & $(652)$ & (998) & (408) & $(1.48)$ & $(0.20)$ & $(0.34)$ & $(24.2)$ & $(41.6)$ & $(24.7)$ \\
\hline \multicolumn{19}{|c|}{ Education (y) } \\
\hline \multirow[t]{2}{*}{$<12$} & 37 & 0.08 & 0.57 & 0.34 & 20.8 & 7.58 & 119 & 1.27 & 76.3 & 11.4 & 19.8 & 6.77 & 0.03 & 0.01 & 0.01 & 0.46 & 0.78 & 0.43 \\
\hline & $(37.4)$ & $(2.72)$ & (18.9) & (10.9\#) & (664) & $(236 \#)$ & (3795) & $(41.1)$ & $(2351 \#)$ & (353\#) & $(628 \#)$ & (210\#) & (1.03) & $(0.19)$ & $(0.29)$ & (14.4) & $(24.2)$ & (13.4\#) \\
\hline \multirow[t]{2}{*}{$\geq 12$} & 62 & 0.10 & 0.62 & 0.40 & 19.8 & 7.76 & 120 & 1.27 & 85.4 & 12.5 & 21.3 & 8.24 & 0.03 & 0.01 & 0.01 & 0.46 & 0.81 & 0.51 \\
\hline & $(62.6)$ & $(3.46)$ & $(20.8)$ & (13.5) & (668) & (259) & $(4050)$ & $(42.5)$ & (2858) & $(417)$ & $(725)$ & $(275)$ & $(0.97)$ & $(0.18)$ & $(0.42)$ & (15.7) & $(27.6)$ & (17.1) \\
\hline
\end{tabular}




\begin{tabular}{|c|c|c|c|c|c|c|c|c|c|c|c|c|c|c|c|c|c|c|}
\hline Parit & & & & & & & & & & & & & & & & & & \\
\hline \multirow[t]{2}{*}{1} & 45 & 0.10 & 0.63 & $0.44 *$ & 22.0 & $9.06 \#$ & 130 & 1.35 & $97.6 \#$ & $14.5^{*}$ & 23.1 & 8.95 & 0.03 & 0.00 & 0.01 & 0.53 & 0.92 & 0.57 \\
\hline & $(45.5)$ & $\left(3.43^{*}\right)$ & (21.3) & $(15.1 * *)$ & $(746 \#)$ & $(302 * *)$ & (4406) & $(45.7)$ & $\left(3255^{*}\right.$ & $\left(485^{* *}\right)$ & $\left(788^{*}\right)$ & (299\#) & $(1.07 \#)$ & $(0.16)$ & $(0.33)$ & $\left(17.9^{*}\right)$ & $\left(31.3^{*}\right)$ & $(19.0 *)$ \\
\hline & & & & & & & & & \multicolumn{10}{|l|}{$*)$} \\
\hline \multirow[t]{2}{*}{2} & 46 & 0.09 & 0.57 & 0.32 & 18.0 & 6.50 & 107 & 1.17 & 68.9 & 10.1 & 18.5 & 6.62 & 0.03 & 0.01 & 0.01 & 0.40 & 0.68 & 0.40 \\
\hline & $(46.5)$ & $(3.10)$ & (18.7) & $(10.3)$ & $(588)$ & (208) & (3490\#) & (38.6) & (2189) & (319) & $(601)$ & $(212)$ & $(0.94)$ & $(0.20)$ & $(0.28)$ & $(12.7)$ & $(21.8)$ & $(12.8)$ \\
\hline \multirow[t]{2}{*}{$\geq 3$} & $8(8.1)$ & 0.07 & 0.66 & 0.33 & 22.3 & 6.89 & 133 & 1.34 & 69.6 & 9.98 & 20.5 & 6.73 & 0.03 & 0.01 & 0.04 & 0.46 & 0.76 & 0.44 \\
\hline & & $(2.27)$ & $(20.6)$ & (10.3) & $(670)$ & (211) & (4087) & $(40.7)$ & (2124) & (307) & $(630)$ & (206) & $(0.84)$ & $(0.24)$ & (1.12) & (14.3) & (23.9) & (13.4) \\
\hline \multicolumn{19}{|c|}{ Time of breastfeeding (month) } \\
\hline \multirow[t]{3}{*}{0} & 10 & $0.08 \#$ & 0.68 & 0.44 & $29.6 \#$ & 11.3\# & 161 & 1.45 & 109 & 17.2 & 28.1 & 9.21 & 0.03 & 0.00 & 0.01 & $0.66 \#$ & 1.10 & 0.61 \\
\hline & $(10.1)$ & $(2.90 * *$ & (23.7) & (15.3) & $(981 *)$ & $(370 *)$ & $(5441)$ & $(48.6)$ & $(3672)$ & $(573 \#)$ & $(963 \#)$ & (318) & (1.09) & $(0.13)$ & $(0.29)$ & $\left(22.0^{*}\right)$ & $(37.2 *)$ & (20.9) \\
\hline & & ) & & & & & & & & & & & & & & & & \\
\hline \multirow[t]{2}{*}{$1-3$} & 20 & 0.11 & 0.61 & 0.38 & 18.5 & 7.02 & 111 & 1.25 & 74.4 & 11.1 & 19.8 & 6.85 & 0.03 & 0.00 & 0.01 & 0.43 & 0.74 & 0.42 \\
\hline & $(20.2)$ & $(3.72)$ & $(20.1)$ & (12.1) & $(621)$ & $(227)$ & $(3690)$ & $(41.5)$ & (2363) & $(351)$ & $(653)$ & (218) & $(0.99)$ & $(0.15)$ & $(0.27)$ & (14.0) & $(23.8)$ & (13.2) \\
\hline
\end{tabular}




\begin{tabular}{|c|c|c|c|c|c|c|c|c|c|c|c|c|c|c|c|c|c|c|}
\hline \multirow[t]{2}{*}{$3-5$} & 40 & 0.10 & 0.57 & 0.38 & 17.7 & 7.05 & 110 & 1.16 & 78.4 & 11.2 & 19.7 & 7.27 & 0.03 & 0.01 & 0.01 & 0.45 & 0.78 & 0.45 \\
\hline & $(40.4)$ & (3.74) & (19.7) & (13.1) & (611) & $(240)$ & (3781) & $(40.2)$ & (2667) & (386) & $(670)$ & $(248)$ & $(0.90)$ & $(0.18)$ & $(0.49)$ & (15.2) & $(26.8)$ & $(15.5)$ \\
\hline \multirow[t]{2}{*}{$\geq 5$} & 24 & 0.07 & 0.61 & 0.36 & 21.8 & 7.97 & 123 & 1.36 & 84.8 & 12.5 & 20.2 & 8.68 & 0.04 & 0.01 & 0.01 & 0.45 & 0.77 & 0.55 \\
\hline & $(24.2)$ & $(2.20)$ & (18.9) & (11.0) & $(661)$ & $(242)$ & (3806) & $(42.2)$ & (2553) & (373) & (632) & $(261)$ & (1.17) & $(0.26)$ & $(0.33)$ & (13.5) & (23.4) & (16.3) \\
\hline \multirow[t]{2}{*}{ Missing } & $5(5.1)$ & 0.06 & 0.64 & 0.34 & 20.3 & 7.07 & 123 & 1.33 & 74.3 & 10.9 & 20.8 & 6.68 & 0.02 & 0.00 & 0.01 & 0.43 & 0.74 & 0.42 \\
\hline & & $(1.81)$ & $(21.3)$ & (11.1) & (689) & (237) & (4139) & $(44.9)$ & $(2452)$ & $(360)$ & (692) & (219) & $(0.66)$ & $(0.10)$ & $(0.21)$ & (14.4) & $(24.8)$ & (13.9) \\
\hline \multicolumn{19}{|l|}{ Ethnicity } \\
\hline \multirow[t]{2}{*}{ German } & 89 & 0.09 & 0.60 & 0.38 & 19.4 & $7.32 *$ & 117 & 1.23 & 81.4 & 11.7 & 20.8 & 7.81 & $0.03 * *$ & 0.01 & 0.01 & 0.46 & 0.80 & 0.49 \\
\hline & (89.9) & (3.23) & (20.4) & (12.8) & $(660)$ & $(246)$ & (3978) & (41.9) & (2703) & (392) & (702\#) & $(258 \#)$ & $(0.93 *)$ & $(0.18)$ & $(0.39)$ & (15.5) & (26.9) & $(16.1 \#)$ \\
\hline Non- & 10 & 0.10 & 0.60 & 0.36 & 27.1 & 11.1 & 138 & 1.57 & 87.1 & 15.2 & 20.0 & 6.60 & 0.06 & 0.01 & 0.01 & 0.47 & 0.78 & 0.43 \\
\hline German & $(10.1)$ & $(2.76)$ & $(16.6)$ & $(9.62)$ & $(727)$ & (292) & $(3747)$ & $(42.8)$ & $(2365)$ & $(405)$ & $(564)$ & (185) & $(1.56)$ & $(0.23)$ & $(0.24)$ & $(13.0)$ & $(21.2)$ & $(11.8)$ \\
\hline \multicolumn{19}{|c|}{ Maternal smoking } \\
\hline \multirow[t]{2}{*}{ No } & 95 & 0.09 & 0.60 & 0.37 & $19.8 \#$ & $7.53 \#$ & 118 & 1.25 & 81.0 & 11.9 & 20.6 & 7.62 & $0.03 * *$ & $0.01 * *$ & 0.01 & 0.46 & 0.79 & 0.48 \\
\hline & $(95.0)$ & $(3.24)$ & $(20.3)$ & $(12.5)$ & $(667)$ & $(250)$ & (3983) & $(42.3)$ & $(2678)$ & $(393)$ & $(694)$ & $(252)$ & $(0.95 \#)$ & $(0.18 \#)$ & $(0.38)$ & $(15.3)$ & $(26.5)$ & $(15.8)$ \\
\hline
\end{tabular}




\begin{tabular}{|c|c|c|c|c|c|c|c|c|c|c|c|c|c|c|c|c|c|c|}
\hline \multirow[t]{2}{*}{ Yes } & \multirow[t]{2}{*}{$4(4.0)$} & 0.09 & 0.68 & 0.52 & 29.3 & 11.6 & 146 & 1.63 & 106 & 17.0 & 24.2 & 9.28 & 0.09 & 0.02 & 0.01 & 0.57 & 0.98 & 0.56 \\
\hline & & $(1.92)$ & (15.2) & (11.8) & $(656)$ & (262) & (3274) & $(36.4)$ & $(2442)$ & (389) & $(552)$ & (214) & (1.90) & $(0.36)$ & $(0.32)$ & (13.1) & $(22.3)$ & (12.8) \\
\hline \multicolumn{19}{|c|}{ Vegetarian } \\
\hline \multirow[t]{2}{*}{ Yes } & 18 & 0.10 & 0.61 & 0.48 & 20.5 & 9.18 & 127 & 1.38 & 100 & 14.8 & 23.4 & 9.10 & 0.03 & 0.01 & 0.01 & 0.53 & 0.95 & 0.56 \\
\hline & (18.2) & $(3.12)$ & (19.0) & (14.1) & (611) & (268) & $(3820)$ & $(42.2)$ & (2909) & $(428)$ & (706) & (267) & $(0.93)$ & $(0.15)$ & $(0.28)$ & (15.1) & $(27.5)$ & (16.3) \\
\hline \multirow[t]{2}{*}{ No } & 81 & 0.09 & 0.60 & 0.35 & 20.1 & 7.37 & 118 & 1.24 & 78.0 & 11.5 & 20.2 & 7.38 & 0.03 & 0.01 & 0.01 & 0.45 & 0.76 & 0.46 \\
\hline & (81.8) & $(3.20)$ & (20.3) & (12.1) & (679) & (247) & (3985) & $(42.0)$ & (2615) & (386) & (685) & $(247)$ & $(1.00)$ & $(0.19)$ & $(0.39)$ & (15.2) & (26.1) & (15.6) \\
\hline \multicolumn{19}{|c|}{ Infant age at sampling (d) } \\
\hline \multirow[t]{3}{*}{$<60$} & 19 & $0.09 *$ & 0.64 & 0.38 & 23.4 & 8.88 & 130 & 1.43 & 85.5 & 13.3 & 22.0 & 7.44 & 0.04 & 0.00 & 0.01 & 0.49 & 0.83 & 0.46 \\
\hline & (19.2) & $(2.84 * *$ & (19.3) & $\left(11.5^{* *}\right)$ & $(705)$ & $(265)$ & (3917) & $(43.1)$ & $(2541)$ & (391) & (664) & $(221)$ & $(1.17)$ & $(0.14)$ & $(0.23 \#)$ & $(15.0)$ & $(25.1)$ & $(13.8)$ \\
\hline & & ) & & & & & & & & & & & & & & & & \\
\hline \multirow[t]{2}{*}{$60-120$} & 37 & 0.11 & 0.56 & 0.39 & 18.6 & 7.16 & 114 & 1.18 & 78.9 & 11.5 & 19.7 & 7.18 & 0.03 & 0.01 & 0.01 & 0.46 & 0.79 & 0.44 \\
\hline & $(37.4)$ & $(3.96)$ & $(20.4)$ & $(14.0)$ & $(680)$ & $(257)$ & $(4115)$ & $(42.9)$ & $(2821)$ & $(412)$ & $(714)$ & $(260)$ & $(0.95)$ & $(0.18)$ & $(0.50)$ & $(16.5)$ & $(28.6)$ & $(16.2)$ \\
\hline
\end{tabular}




\begin{tabular}{|c|c|c|c|c|c|c|c|c|c|c|c|c|c|c|c|c|c|c|}
\hline $120-180$ & 28 & 0.08 & 0.63 & 0.42 & 19.8 & 8.15 & 121 & 1.27 & 92.7 & 13.5 & 22.0 & 9.25 & 0.03 & 0.01 & 0.01 & 0.48 & 0.85 & 0.59 \\
\hline & $(28.3)$ & $(3.09)$ & $(21.3)$ & (13.7) & $(647)$ & $(260)$ & (3985) & $(41.5)$ & $(2965)$ & $(431)$ & (732) & (294) & $(0.91)$ & $(0.17)$ & $(0.33)$ & (15.4) & $(27.6)$ & (18.5) \\
\hline \multirow[t]{2}{*}{$\geq 180$} & 14 & 0.06 & 0.58 & 0.26 & 20.2 & 6.24 & 116 & 1.29 & 65.3 & 9.14 & 19.7 & 6.51 & 0.03 & 0.01 & 0.01 & 0.41 & 0.68 & 0.41 \\
\hline & $(14.1)$ & $(1.88)$ & $(17.8)$ & $(7.80)$ & (610) & (188) & $(3505)$ & (39.9) & (1901) & $(270)$ & $(580)$ & (190) & $(1.00)$ & $(0.27)$ & $(0.34)$ & (11.8) & (19.9) & (12.0) \\
\hline \multicolumn{19}{|c|}{ Infant gender } \\
\hline \multirow[t]{2}{*}{ Female } & 47 & 0.09 & 0.65 & 0.42 & 22.2 & 8.65 & 130 & 1.39 & $94.7 \#$ & $13.9 \#$ & 22.9 & $9.01 \#$ & $0.04 *$ & 0.01 & 0.01 & $0.51 \#$ & $0.88 \#$ & $0.57 *$ \\
\hline & $(47.5)$ & $(2.84)$ & $(20.0)$ & (12.8) & (677) & (262) & (4008) & $(42.2)$ & (2877) & $(420)$ & (709) & $(272)$ & $(1.10)$ & $(0.18)$ & (0.29\#) & $(15.7)$ & $(27.3)$ & (17.1) \\
\hline \multirow[t]{2}{*}{ Male } & 48 & 0.09 & 0.56 & 0.35 & 18.5 & 6.92 & 111 & 1.17 & 71.3 & 10.6 & 18.9 & 6.59 & 0.03 & 0.01 & 0.01 & 0.43 & 0.73 & 0.41 \\
\hline & $(48.5)$ & $(3.53)$ & (20.2) & (12.4) & $(656)$ & (241) & (3910) & (41.9) & (2483) & $(371)$ & $(667)$ & (233) & $(0.91)$ & $(0.19)$ & $(0.47)$ & (14.7) & $(25.6)$ & (14.5) \\
\hline \multirow[t]{2}{*}{ Missing } & $4(4.0)$ & 0.08 & 0.50 & 0.28 & 16.6 & 5.86 & 98.0 & 1.05 & 60.8 & 8.85 & 17.5 & 5.39 & 0.02 & 0.00 & 0.01 & 0.36 & 0.60 & 0.34 \\
\hline & & (3.06) & (18.3) & (10.8) & (669) & (237) & (3863) & $(41.0)$ & (2440) & (354) & $(701)$ & $(221)$ & $(0.60)$ & $(0.15)$ & $(0.21)$ & (14.4) & $(24.2)$ & (13.8) \\
\hline
\end{tabular}


Table S12 Correlations between demographic characteristics and PCDD/Fs, 2 mono-ortho PCBs, $\Sigma$ non-ortho PCBs, $\Sigma$ PBDEs, and $\Sigma$ POPs in breast milk. Comparisons were performed using Welch t-test or analysis of variance (ANOVA). Chemical concentrations were expressed as $\mathrm{pg} / \mathrm{mL}$ milk and $\mathrm{pg} / \mathrm{g}$ lw (in the parentheses).

\begin{tabular}{|c|c|c|c|c|c|c|c|c|c|c|c|c|}
\hline & $\mathrm{N}(\%)$ & $1,2,3,6,7,8-$ & $1,2,3,4,6,7$ & OCDD & $2,3,4,7,8-$ & $1,2,3,6,7,8-$ & $\Sigma \mathrm{PBDE}$ & $\Sigma \mathrm{PCB}$ & $\Sigma \mathrm{PCDDF}$ & $\Sigma$ POPs & Smono- & इnon-ortho \\
\hline & & $\mathrm{HxCDD}$ & 8-HpCDD & & $\mathrm{PeCDF}$ & $\mathrm{HxCDF}$ & & & & & ortho PCBs & PCBs \\
\hline \multicolumn{13}{|c|}{ Maternal age (y) } \\
\hline \multirow[t]{2}{*}{$\leq 29$} & $15(15.2)$ & $0.06^{*}$ & $0.06(1.91)$ & $0.40(13.1)$ & $0.11(3.45)$ & $0.02(0.66)$ & $285(9058)$ & $284(8682)$ & $0.65 *(20.9 * *)$ & $569(17761)$ & $281(8592)$ & $1.03(32.0)$ \\
\hline & & $\left(1.86^{*}\right)$ & & & & & & & & & & \\
\hline $30-39$ & $75(75.8)$ & $0.09(2.96)$ & $0.10(3.22)$ & $0.67(22.3)$ & $0.12(4.04)$ & $0.03(1.04)$ & 144 (5597) & $264(8900)$ & $1.01(33.6)$ & $408(14531)$ & $261(8807)$ & $1.06(36.2)$ \\
\hline \multirow[t]{2}{*}{$\geq 40$} & $9(9.1)$ & $0.14(4.06)$ & $0.09(3.02)$ & $0.79(23.5)$ & $0.15(4.77)$ & $0.03(0.90)$ & 54.7 & $342(10462)$ & $1.21(36.3)$ & $398(12033)$ & $339(10352)$ & $1.21(37.5)$ \\
\hline & & & & & & & $(1535)$ & & & & & \\
\hline \multicolumn{13}{|c|}{ Maternal BMI $\left(\mathrm{kg} / \mathrm{m}^{2}\right)$} \\
\hline \multirow[t]{2}{*}{$<24.9$} & $66(66.7)$ & $0.09(3.06)$ & 0.09 & $0.61(19.9)$ & $0.12 \#$ & $0.03 \#$ & $118(4510)$ & $280(9413 *)$ & $0.94(31.2)$ & 399 (13954) & $277(9314 *)$ & $1.08(36.8 \#)$ \\
\hline & & & $(2.99 *)$ & & $(4.19 * *)$ & $(1.01 \#)$ & & & & & & \\
\hline
\end{tabular}




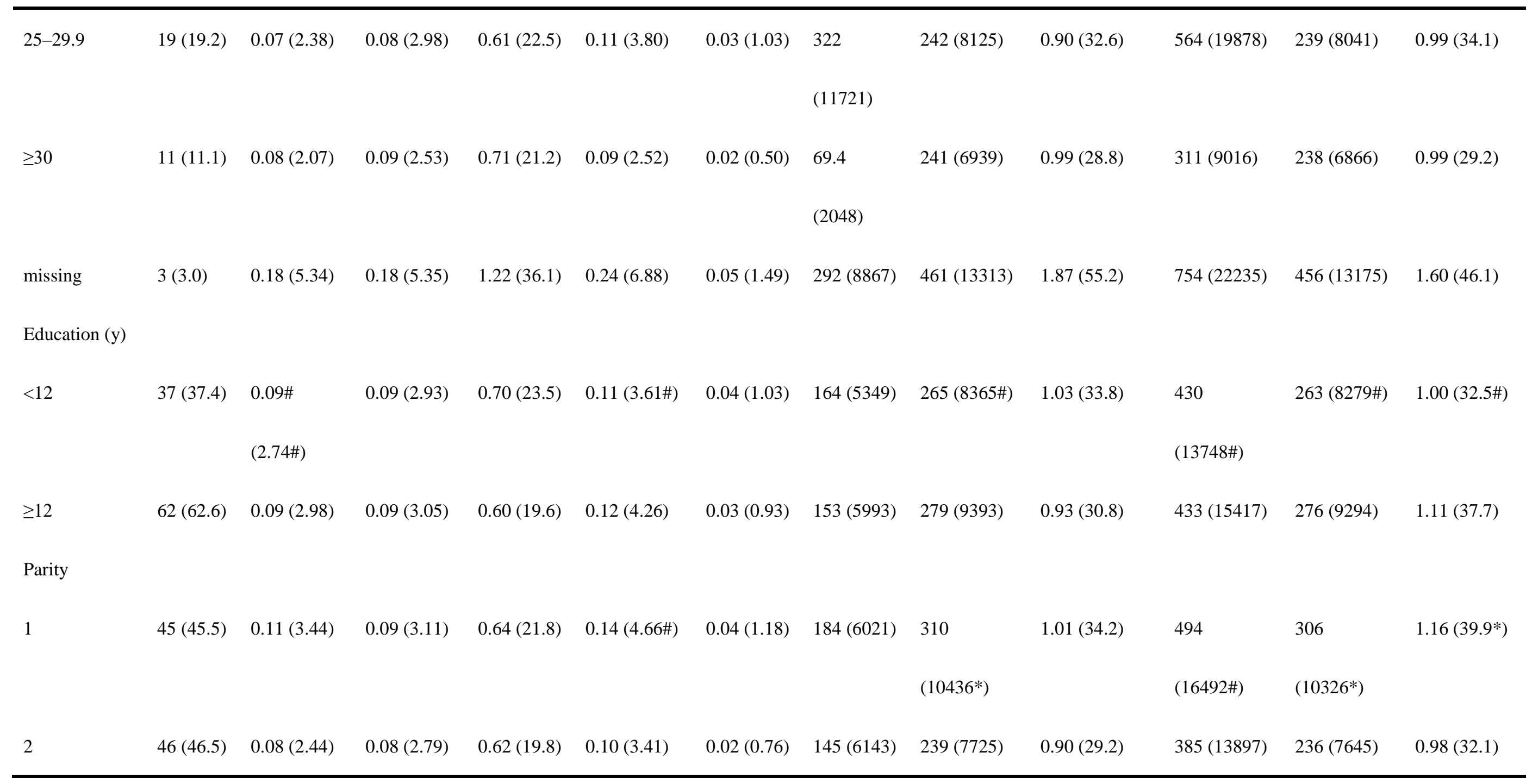




\begin{tabular}{|c|c|c|c|c|c|c|c|c|c|c|c|c|}
\hline$\geq 3$ & $8(8.1)$ & $0.07(2.41)$ & $0.12(3.66)$ & $0.76(23.9)$ & $0.12(3.85)$ & $0.03(1.02)$ & $\begin{array}{l}73.7 \\
(1996)\end{array}$ & $273(8362)$ & $1.10(34.9)$ & 348 (10393) & $271(8275)$ & $1.06(33.2)$ \\
\hline \multicolumn{13}{|c|}{ Time of breastfeeding (month) } \\
\hline 0 & $10(10.1)$ & $0.09(3.30)$ & $0.07(2.79)$ & $0.67(24.4)$ & $0.16(5.62)$ & $0.03(0.99)$ & $\begin{array}{l}46.4^{*} \\
(1557 *)\end{array}$ & $\begin{array}{l}370 \\
(12489 \#)\end{array}$ & $1.02(37.1)$ & $\begin{array}{l}417 \\
(14083 \#)\end{array}$ & $\begin{array}{l}366 \\
(12366 \#)\end{array}$ & $1.20(41.9)$ \\
\hline $1-3$ & $20(20.2)$ & $0.09(2.85)$ & $0.08(2.73)$ & $0.56(18.8)$ & $0.12(4.18)$ & $0.03(0.91)$ & $\begin{array}{l}424 \\
\text { (13914) }\end{array}$ & 253 (8253) & 0.88 (29.4) & 677 (22197) & $250(8165)$ & $1.10(35.9)$ \\
\hline $3-5$ & $40(40.4)$ & $0.10(3.10)$ & $0.09(3.12)$ & $0.63(21.1)$ & $0.11(3.88)$ & $0.04(1.15)$ & $102(4980)$ & 256 (8739) & $0.97(32.3)$ & $359(13750)$ & $253(8643)$ & $1.04(36.5)$ \\
\hline$\geq 5$ & $24(24.2)$ & $0.08(2.38)$ & $0.11(3.34)$ & $0.74(22.2)$ & $0.11(3.39)$ & $0.02(0.69)$ & $\begin{array}{l}93.9 \\
(2833)\end{array}$ & 283 (8658) & $1.05(32.0)$ & 378 (11522) & $280(8571)$ & $1.04(32.1)$ \\
\hline Missing & $5(5.1)$ & $0.09(2.97)$ & $0.06(2.05)$ & $0.54(17.7)$ & $0.13(4.23)$ & $0.03(1.03)$ & $\begin{array}{l}49.3 \\
(1696)\end{array}$ & $267(8921)$ & $0.85(28.0)$ & $317(10645)$ & $264(8833)$ & $1.04(34.2)$ \\
\hline Ethnicity & & & & & & & & & & & & \\
\hline
\end{tabular}




\begin{tabular}{|c|c|c|c|c|c|c|c|c|c|c|c|c|}
\hline German & $89(89.9)$ & $0.09(3.01 *)$ & $0.09(3.14)$ & 0.66 & $0.12(4.14 *)$ & $0.03(1.00)$ & 155 (5827) & 270 (90778) & $0.99\left(33.3^{*}\right)$ & $426(14937)$ & 267 (8981) & $1.07(36.5)$ \\
\hline & & & & $(22.0 *)$ & & & & & & & & \\
\hline Non-German & $10(10.1)$ & $0.07(1.82)$ & $0.07(1.79)$ & $0.47(12.5)$ & $0.11(2.89)$ & $0.03(0.73)$ & $175(5088)$ & 309 (8405) & $0.75(19.8)$ & 485 (13512) & 307 (8328) & $1.06(28.9)$ \\
\hline \multicolumn{13}{|c|}{ Maternal somking } \\
\hline \multirow[t]{2}{*}{ No } & $95(95.0)$ & $0.09(2.90)$ & 0.09 & $0.64(21.2)$ & $0.12(4.03)$ & $0.03(0.97)$ & 158 (5889) & $271(9056)$ & $0.96(32.2)$ & 430 (14977) & $268(8960)$ & $1.06(36.0)$ \\
\hline & & & $(3.07 \#)$ & & & & & & & & & \\
\hline Yes & $4(4.0)$ & $0.12(2.70)$ & $0.08(1.59)$ & $0.71(15.9)$ & $0.17(3.73)$ & $0.04(0.87)$ & $122(2508)$ & 349 (7904) & $1.12(24.8)$ & $472(10438)$ & 345 (7825) & $1.29(28.9)$ \\
\hline \multicolumn{13}{|l|}{ Vegetarian } \\
\hline \multirow[t]{2}{*}{ Yes } & $18(18.2)$ & $0.15^{* *}$ & $0.09(2.86)$ & $0.65(19.0)$ & $0.15 \#(4.47)$ & $0.06^{*}$ & $256^{*}$ & 308 (9147) & $1.10(31.9)$ & $568^{*}$ & $305(9050)$ & $1.19(36.2)$ \\
\hline & & $(4.07 *)$ & & & & $(1.49 *)$ & (10436\#) & & & (19614\#) & & \\
\hline No & $81(81.8)$ & $0.08(2.63)$ & $0.09(3.04)$ & $0.64(21.5)$ & $0.11(3.91)$ & $0.02(0.85)$ & $134(4712)$ & 266 (8979) & $0.94(31.9)$ & $401(13722)$ & $263(8884)$ & $1.04(35.6)$ \\
\hline \multicolumn{13}{|c|}{ Infant age at sampling } \\
\hline$<60$ & $19(19.2)$ & $0.09(2.77)$ & $0.09(2.87)$ & $0.71(20.7)$ & $0.12(3.79)$ & $0.03(0.78)$ & $455^{*}$ & 295 (8837) & $1.05(31.0)$ & $751^{*}$ & $292(8748)$ & $1.12(33.7 \#)$ \\
\hline & & & & & & & (14293\#) & & & $(23161 \#)$ & & \\
\hline
\end{tabular}




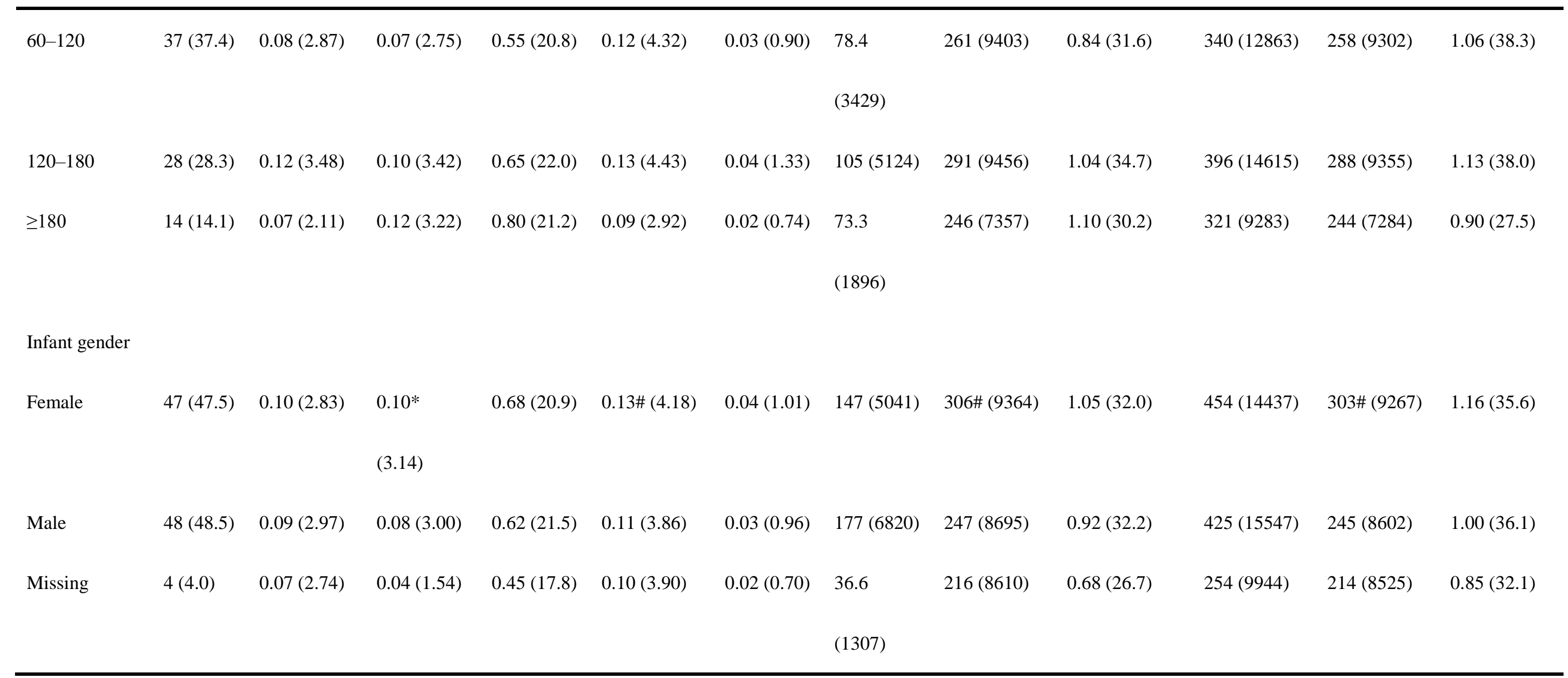


Table S13 Crude associations ( $\beta$ coefficient, 95\% CI) between POPs (DF $\geq 80 \%$ ) and THs in human breast milk, single pollutant models.

\begin{tabular}{|c|c|c|c|c|c|c|c|}
\hline POPs & & $\mathrm{TT}_{4} \beta(95 \% \mathrm{CI})$ & $p$-value & $\mathrm{TT}_{3} \beta(95 \% \mathrm{CI})$ & $p$-value & $\operatorname{TrT}_{3} \beta(95 \% \mathrm{CI})$ & $p$-value \\
\hline \multirow{3}{*}{ BDE-28 } & 1 & Reference & & Reference & & Reference & \\
\hline & 2 & $-0.05(-0.17-0.07)$ & 0.39 & $-0.02(-0.12-0.09)$ & 0.76 & $-0.12(-0.32-0.08)$ & 0.25 \\
\hline & 3 & $-0.10(-0.22-0.03)$ & 0.12 & $-0.09(-0.19-0.01)$ & 0.09 & $-0.13(-0.33-0.07)$ & 0.19 \\
\hline \multirow{3}{*}{ BDE-47 } & 1 & Reference & & Reference & & Reference & \\
\hline & 2 & $-0.07(-0.19-0.05)$ & 0.23 & $-0.01(-0.11-0.09)$ & 0.82 & $-0.22(-0.42--0.03)$ & 0.03 \\
\hline & 3 & $-0.12(-0.24-0.00)$ & 0.05 & $-0.11(-0.21-0.00)$ & 0.04 & $-0.23(-0.42--0.03)$ & 0.02 \\
\hline \multirow{3}{*}{ BDE-99 } & 1 & Reference & & Reference & & Reference & \\
\hline & 2 & $-0.12(-0.24-0.00)$ & 0.05 & $0.02(-0.09-0.12)$ & 0.75 & $-0.19(-0.39-0.00)$ & 0.05 \\
\hline & 3 & $-0.16(-0.27--0.04)$ & 0.01 & $-0.08(-0.18-0.02)$ & 0.13 & $-0.31(-0.50--0.11)$ & 0.002 \\
\hline \multirow{3}{*}{ BDE-100 } & 1 & Reference & & Reference & & Reference & \\
\hline & 2 & $-0.06(-0.18-0.06)$ & 0.33 & $-0.06(-0.16-0.05)$ & 0.28 & $-0.13(-0.33-0.07)$ & 0.20 \\
\hline & 3 & $-0.12(-0.24-0.01)$ & 0.06 & $-0.10(-0.21-0.00)$ & 0.04 & $-0.22(-0.42--0.02)$ & 0.03 \\
\hline \multirow{3}{*}{ BDE-153 } & 1 & Reference & & Reference & & Reference & \\
\hline & 2 & $-0.01(-0.13-0.11)$ & 0.90 & $-0.05(-0.15-0.05)$ & 0.34 & $-0.02(-0.22-0.18)$ & 0.86 \\
\hline & 3 & $-0.07(-0.19-0.05)$ & 0.26 & $-0.10(-0.20-0.01)$ & 0.06 & $-0.20(-0.40-0.00)$ & 0.05 \\
\hline \multirow{3}{*}{ BDE-154 } & 1 & Reference & & Reference & & Reference & \\
\hline & 2 & $-0.11(-0.23-0.01)$ & 0.07 & $-0.01(-0.12-0.09)$ & 0.78 & $-0.09(-0.28-0.11)$ & 0.39 \\
\hline & 3 & $-0.16(-0.28--0.05)$ & 0.01 & $-0.07(-0.17-0.03)$ & 0.19 & $-0.22(-0.41--0.02)$ & 0.03 \\
\hline BDE-183 & 1 & Reference & & Reference & & Reference & \\
\hline
\end{tabular}




\begin{tabular}{|c|c|c|c|c|c|c|c|}
\hline & 2 & $-0.03(-0.15-0.10)$ & 0.67 & $0.00(-0.11-0.10)$ & 0.95 & $-0.14(-0.34-0.05)$ & 0.15 \\
\hline & 3 & $-0.06(-0.19-0.06)$ & 0.30 & $-0.05(-0.15-0.06)$ & 0.37 & $-0.24(-0.44--0.05)$ & 0.02 \\
\hline \multirow{4}{*}{ BDE-196 } & 1 & Reference & & Reference & & Reference & \\
\hline & 2 & $-0.11(-0.23-0.01)$ & 0.07 & $-0.04(-0.14-0.06)$ & 0.47 & $-0.14(-0.34-0.06)$ & 0.16 \\
\hline & 3 & $-0.14(-0.26--0.02)$ & 0.03 & $-0.10(-0.20-0.00)$ & 0.05 & $-0.20(-0.40-0.00)$ & 0.05 \\
\hline & 1 & Reference & & Reference & & Reference & \\
\hline \multirow[t]{3}{*}{ BDE-197 } & 2 & $-0.06(-0.18-0.06)$ & 0.35 & $-0.11(-0.21--0.01)$ & 0.04 & $-0.10(-0.30-0.10)$ & 0.31 \\
\hline & 3 & $-0.05(-0.18-0.07)$ & 0.38 & $-0.09(-0.19-0.01)$ & 0.07 & $-0.16(-0.36-0.04)$ & 0.11 \\
\hline & 1 & Reference & & Reference & & Reference & \\
\hline \multirow[t]{3}{*}{ BDE-203 } & 2 & $-0.02(-0.14-0.10)$ & 0.74 & $-0.02(-0.12-0.08)$ & 0.68 & $-0.13(-0.32-0.07)$ & 0.20 \\
\hline & 3 & $-0.13(-0.25--0.01)$ & 0.04 & $-0.17(-0.27--0.07)$ & 0.001 & $-0.25(-0.45--0.05)$ & 0.01 \\
\hline & 1 & Reference & & Reference & & Reference & \\
\hline \multirow[t]{3}{*}{ BDE-206 } & 2 & $-0.03(-0.15-0.09)$ & 0.62 & $0.00(-0.10-0.11)$ & 0.93 & $0.01(-0.19-0.21)$ & 0.93 \\
\hline & 3 & $-0.05(-0.17-0.08)$ & 0.45 & $-0.02(-0.12-0.09)$ & 0.74 & $0.01(-0.19-0.22)$ & 0.89 \\
\hline & 1 & Reference & & Reference & & Reference & \\
\hline \multirow[t]{3}{*}{ BDE-207 } & 2 & $-0.06(-0.19-0.06)$ & 0.29 & $-0.02(-0.12-0.08)$ & 0.65 & $-0.07(-0.27-0.13)$ & 0.48 \\
\hline & 3 & $-0.10(-0.22-0.03)$ & 0.12 & $-0.14(-0.24--0.04)$ & 0.01 & $-0.15(-0.35-0.05)$ & 0.14 \\
\hline & 1 & Reference & & Reference & & Reference & \\
\hline \multirow[t]{3}{*}{ BDE-208 } & 2 & $-0.07(-0.20-0.05)$ & 0.23 & $-0.07(-0.17-0.03)$ & 0.18 & $-0.04(-0.24-0.16)$ & 0.71 \\
\hline & 3 & $-0.07(-0.19-0.06)$ & 0.29 & $-0.12(-0.22--0.02)$ & 0.02 & $-0.09(-0.30-0.11)$ & 0.36 \\
\hline & 1 & Reference & & Reference & & Reference & \\
\hline \multicolumn{4}{|l|}{ BDE-209 } & $002(009012)$ & 060 & $015(005035)$ & 015 \\
\hline
\end{tabular}




\begin{tabular}{|c|c|c|c|c|c|c|c|}
\hline & 3 & $0.03(-0.09-0.16)$ & 0.59 & $-0.02(-0.12-0.08)$ & 0.71 & $0.02(-0.18-0.22)$ & 0.87 \\
\hline \multirow{3}{*}{$\Sigma$ PBDEs } & 1 & Reference & & Reference & & Reference & \\
\hline & 2 & $-0.11(-0.23-0.01)$ & 0.08 & $-0.07(-0.17-0.04)$ & 0.20 & $-0.19(-0.39-0.01)$ & 0.06 \\
\hline & 3 & $-0.11(-0.23-0.01)$ & 0.07 & $-0.12(-0.22--0.02)$ & 0.02 & $-0.13(-0.33-0.07)$ & 0.19 \\
\hline \multirow{3}{*}{ PCB-28 } & 1 & Reference & & Reference & & Reference & \\
\hline & 2 & $0.03(-0.09-0.15)$ & 0.65 & $-0.08(-0.18-0.02)$ & 0.76 & $-0.02(-0.22-0.19)$ & 0.87 \\
\hline & 3 & $0.10(-0.02-0.22)$ & 0.10 & $0.01(-0.09-0.11)$ & 0.09 & $0.07(-0.13-0.27)$ & 0.49 \\
\hline \multirow{3}{*}{ PCB-52 } & 1 & Reference & & Reference & & Reference & \\
\hline & 2 & $0.02(-0.10-0.14)$ & 0.77 & $-0.04(-0.14-0.06)$ & 0.45 & $-0.01(-0.21-0.19)$ & 0.93 \\
\hline & 3 & $0.05(-0.07-0.17)$ & 0.40 & $-0.04(-0.14-0.06)$ & 0.46 & $0.03(-0.17-0.23)$ & 0.78 \\
\hline \multirow{3}{*}{ PCB-77 } & 1 & Reference & & Reference & & Reference & \\
\hline & 2 & $-0.04(-0.17-0.08)$ & 0.47 & $-0.09(-0.19-0.02)$ & 0.10 & $0.01(-0.19-0.21)$ & 0.93 \\
\hline & 3 & $-0.09(-0.21-0.03)$ & 0.14 & $-0.05(-0.16-0.05)$ & 0.31 & $-0.12(-0.32-0.08)$ & 0.23 \\
\hline \multirow{3}{*}{ PCB-101 } & 1 & Reference & & Reference & & Reference & \\
\hline & 2 & $-0.02(-0.14-0.10)$ & 0.72 & $-0.07(-0.18-0.03)$ & 0.15 & $-0.05(-0.26-0.15)$ & 0.60 \\
\hline & 3 & $-0.01(-0.14-0.11)$ & 0.83 & $-0.11(-0.21--0.01)$ & 0.03 & $-0.09(-0.29-0.11)$ & 0.37 \\
\hline \multirow{3}{*}{ PCB-105 } & 1 & Reference & & Reference & & Reference & \\
\hline & 2 & $-0.08(-0.20-0.04)$ & 0.18 & $-0.07(-0.17-0.03)$ & 0.19 & $-0.07(-0.27-0.14)$ & 0.52 \\
\hline & 3 & $-0.07(-0.19-0.06)$ & 0.29 & $-0.08(-0.18-0.03)$ & 0.14 & $0.02(-0.18-0.22)$ & 0.82 \\
\hline \multirow{3}{*}{ PCB-114 } & 1 & Reference & & Reference & & Reference & \\
\hline & 2 & $0.00(-0.12-0.12)$ & 0.99 & $-0.02(-0.13-0.08)$ & 0.68 & $0.00(-0.20-0.20)$ & 0.98 \\
\hline & 3 & $-0.07(-0.19-0.06)$ & 0.29 & $-0.07(-0.17-0.04)$ & 0.20 & $-0.07(-0.27-0.13)$ & 0.49 \\
\hline
\end{tabular}




\begin{tabular}{|c|c|c|c|c|c|c|c|}
\hline \multirow{3}{*}{ PCB-118 } & 1 & \multicolumn{2}{|l|}{ Reference } & \multicolumn{2}{|l|}{ Reference } & \multicolumn{2}{|l|}{ Reference } \\
\hline & 2 & $-0.05(-0.18-0.07)$ & 0.38 & $-0.06(-0.17-0.04)$ & 0.23 & $-0.11(-0.31-0.09)$ & 0.29 \\
\hline & 3 & $-0.06(-0.18-0.06)$ & 0.32 & $-0.08(-0.19-0.02)$ & 0.11 & $-0.03(-0.23-0.18)$ & 0.80 \\
\hline \multirow{3}{*}{ PCB-123 } & 1 & Reference & & Reference & \multicolumn{3}{|c|}{ Reference } \\
\hline & 2 & $0.03(-0.09-0.15)$ & 0.66 & $0.03(-0.07-0.13)$ & 0.56 & $0.08(-0.12-0.29)$ & 0.41 \\
\hline & 3 & $-0.02(-0.14-0.10)$ & 0.72 & $-0.02(-0.13-0.08)$ & 0.68 & $0.06(-0.14-0.26)$ & 0.57 \\
\hline \multirow{3}{*}{ PCB-126 } & 1 & Reference & & Reference & \multicolumn{3}{|c|}{ Reference } \\
\hline & 2 & $0.08(-0.04-0.21)$ & 0.17 & $0.01(-0.09-0.11)$ & 0.86 & $0.15(-0.05-0.35)$ & 0.13 \\
\hline & 3 & $-0.02(-0.14-0.10)$ & 0.71 & $0.00(-0.11-0.10)$ & 0.96 & $0.06(-0.14-0.26)$ & 0.57 \\
\hline \multirow{3}{*}{ PCB-138 } & 1 & Reference & & Reference & \multicolumn{3}{|c|}{ Reference } \\
\hline & 2 & $0.00(-0.13-0.12)$ & 0.94 & $-0.06(-0.16-0.04)$ & 0.24 & $-0.03(-0.23-0.17)$ & 0.78 \\
\hline & 3 & $-0.06(-0.18-0.06)$ & 0.33 & $-0.08(-0.18-0.03)$ & 0.14 & $-0.08(-0.28-0.12)$ & 0.42 \\
\hline \multirow{3}{*}{ PCB-153 } & 1 & Reference & & Reference & \multicolumn{3}{|c|}{ Reference } \\
\hline & 2 & $0.00(-0.12-0.12)$ & 0.96 & $-0.05(-0.16-0.05)$ & 0.30 & $-0.05(-0.25-0.15)$ & 0.62 \\
\hline & 3 & $-0.09(-0.21-0.04)$ & 0.17 & $-0.09(-0.19-0.01)$ & 0.08 & $-0.11(-0.31-0.10)$ & 0.30 \\
\hline \multirow{3}{*}{ PCB-156 } & 1 & Reference & & Reference & \multicolumn{3}{|c|}{ Reference } \\
\hline & 2 & $-0.10(-0.23-0.02)$ & 0.09 & $-0.10(-0.21-0.00)$ & 0.04 & $-0.09(-0.29-0.11)$ & 0.37 \\
\hline & 3 & $-0.07(-0.19-0.05)$ & 0.25 & $-0.09(-0.19-0.01)$ & 0.07 & $-0.13(-0.32-0.07)$ & 0.22 \\
\hline \multirow{3}{*}{ PCB-157 } & 1 & Reference & & Reference & \multicolumn{3}{|c|}{ Reference } \\
\hline & 2 & $-0.02(-0.14-0.10)$ & 0.73 & $-0.06(-0.16-0.04)$ & 0.23 & $0.00(-0.20-0.20)$ & 0.97 \\
\hline & 3 & $-0.05(-0.18-0.07)$ & 0.39 & $-0.08(-0.19-0.02)$ & 0.11 & $-0.11(-0.31-0.09)$ & 0.26 \\
\hline PCB-167 & 1 & Reference & & Reference & \multicolumn{3}{|c|}{ Reference } \\
\hline
\end{tabular}




\begin{tabular}{|c|c|c|c|c|c|c|c|}
\hline & 2 & $-0.06(-0.18-0.06)$ & 0.32 & $-0.08(-0.18-0.02)$ & 0.12 & $-0.06(-0.26-0.14)$ & 0.56 \\
\hline & 3 & $-0.10(-0.22-0.02)$ & 0.11 & $-0.09(-0.19-0.01)$ & 0.08 & $-0.08(-0.28-0.12)$ & 0.45 \\
\hline \multirow{4}{*}{ PCB-169 } & 1 & Reference & & Reference & & Reference & \\
\hline & 2 & $0.00(-0.11-0.12)$ & 0.94 & $-0.08(-0.18-0.02)$ & 0.11 & $-0.02(-0.21-0.18)$ & 0.11 \\
\hline & 3 & $-0.13(-0.25--0.01)$ & 0.03 & $-0.14(-0.24--0.05)$ & 0.005 & $-0.18(-0.38-0.02)$ & 0.15 \\
\hline & 1 & Reference & & Reference & & Reference & \\
\hline \multirow[t]{3}{*}{ PCB-180 } & 2 & $-0.06(-0.19-0.06)$ & 0.31 & $-0.10(-0.20-0.00)$ & 0.06 & $-0.11(-0.31-0.09)$ & 0.28 \\
\hline & 3 & $-0.07(-0.19-0.05)$ & 0.26 & $-0.08(-0.19-0.02)$ & 0.10 & $-0.14(-0.33-0.06)$ & 0.18 \\
\hline & 1 & Reference & & Reference & & Reference & \\
\hline \multirow[t]{3}{*}{ PCB-189 } & 2 & $-0.08(-0.20-0.04)$ & 0.19 & $-0.10(-0.21-0.00)$ & 0.05 & $-0.16(-0.36-0.04)$ & 0.11 \\
\hline & 3 & $-0.06(-0.18-0.06)$ & 0.31 & $-0.08(-0.18-0.02)$ & 0.11 & $-0.15(-0.34-0.05)$ & 0.15 \\
\hline & 1 & Reference & & Reference & & Reference & \\
\hline \multirow[t]{3}{*}{$\Sigma \mathrm{PCBs}$} & 2 & $-0.03(-0.15-0.10)$ & 0.67 & $-0.06(-0.16-0.04)$ & 0.25 & $0.02(-0.19-0.22)$ & 0.87 \\
\hline & 3 & $-0.07(-0.19-0.05)$ & 0.25 & $-0.08(-0.18-0.03)$ & 0.14 & $-0.06(-0.27-0.14)$ & 0.53 \\
\hline & 1 & Reference & & Reference & & Reference & \\
\hline \multirow[t]{3}{*}{ 2,3,4,7,8-PeCDF } & 2 & $-0.04(-0.16-0.08)$ & 0.52 & $0.03(-0.07-0.13)$ & 0.57 & $-0.04(-0.24-0.16)$ & 0.67 \\
\hline & 3 & $-0.08(-0.20-0.05)$ & 0.22 & $-0.08(-0.18-0.03)$ & 0.14 & $-0.11(-0.32-0.09)$ & 0.26 \\
\hline & 1 & Reference & & Reference & & Reference & \\
\hline \multirow[t]{3}{*}{ 1,2,3,6,7,8-HxCDF } & 2 & $0.04(-0.08-0.16)$ & 0.50 & $-0.07(-0.17-0.03)$ & 0.18 & $0.00(-0.20-0.20)$ & 0.99 \\
\hline & 3 & $0.00(-0.13-0.12)$ & 0.96 & $-0.06(-0.16-0.05)$ & 0.28 & $-0.04(-0.24-0.16)$ & 0.69 \\
\hline & 1 & Reference & & Reference & & Reference & \\
\hline \multicolumn{8}{|l|}{ 1,2,3,6,7,8-HxCDD } \\
\hline & 2 & $-0.04(-0.16-0.08)$ & 0.52 & $-0.08(-0.18-0.02)$ & 0.12 & $-0.11(-0.31-0.09)$ & 0.29 \\
\hline
\end{tabular}




\begin{tabular}{|c|c|c|c|c|c|c|c|}
\hline & 3 & $-0.13(-0.25--0.01)$ & 0.03 & $-0.09(-0.19-0.01)$ & 0.09 & $-0.15(-0.35-0.05)$ & 0.14 \\
\hline \multirow{3}{*}{ 1,2,3,4,6,7,8-HpCDD } & 1 & Reference & & Reference & & Reference & \\
\hline & 2 & $-0.05(-0.17-0.07)$ & 0.40 & $-0.04(-0.14-0.07)$ & 0.49 & $-0.12(-0.32-0.08)$ & 0.23 \\
\hline & 3 & $-0.11(-0.23-0.01)$ & 0.08 & $-0.10(-0.20-0.01)$ & 0.07 & $-0.24(-0.44--0.05)$ & 0.02 \\
\hline \multirow{3}{*}{ OCDD } & 1 & Reference & & Reference & & Reference & \\
\hline & 2 & $-0.04(-0.16-0.08)$ & 0.55 & $-0.03(-0.13-0.07)$ & 0.59 & $-0.12(-0.32-0.08)$ & 0.23 \\
\hline & 3 & $-0.10(-0.22-0.02)$ & 0.09 & $-0.15(-0.25--0.06)$ & 0.002 & $-0.11(-0.31-0.09)$ & 0.29 \\
\hline \multirow{3}{*}{$\Sigma$ PCDDFs } & 1 & Reference & & Reference & & Reference & \\
\hline & 2 & $-0.03(-0.15-0.09)$ & 0.57 & $-0.07(-0.17-0.03)$ & 0.18 & $-0.11(-0.30-0.09)$ & 0.29 \\
\hline & 3 & $-0.11(-0.23-0.01)$ & 0.07 & $-0.14(-0.24-0.04)$ & 0.01 & $-0.18(-0.38-0.01)$ & 0.07 \\
\hline \multirow{3}{*}{$\Sigma$ POPs } & 1 & Reference & & Reference & & Reference & \\
\hline & 2 & $0.01(-0.11-0.13)$ & 0.86 & $-0.01(-0.11-0.09)$ & 0.84 & $-0.10(-0.30-0.10)$ & 0.33 \\
\hline & 3 & $-0.05(-0.18-0.07)$ & 0.39 & $-0.11(-0.21--0.01)$ & 0.03 & $-0.01(-0.21-0.19)$ & 0.95 \\
\hline
\end{tabular}


Table S14 Summary of the factor loadings for five factors using principal component analysis with varimax rotation.

\begin{tabular}{|c|c|c|c|c|c|c|c|c|}
\hline & Factor 1 & Factor 2 & Factor 3 & Factor 4 & Factor 5 & h2 & u2 & com \\
\hline BDE-28 & 0.23 & 0.12 & 0.57 & 0.4 & -0.01 & 0.55 & 0.448 & 2.3 \\
\hline BDE-47 & -0.11 & -0.03 & 0.88 & 0.14 & 0.02 & 0.81 & 0.189 & 1.1 \\
\hline BDE-99 & -0.13 & 0.02 & 0.86 & 0.13 & -0.05 & 0.78 & 0.217 & 1.1 \\
\hline BDE-100 & 0.01 & -0.03 & 0.86 & 0.22 & 0.12 & 0.81 & 0.192 & 1.2 \\
\hline BDE-153 & 0.62 & 0.08 & 0.51 & -0.01 & -0.03 & 0.65 & 0.351 & 2 \\
\hline BDE-154 & 0.06 & -0.08 & 0.78 & -0.01 & 0.04 & 0.62 & 0.381 & 1 \\
\hline BDE-183 & 0.22 & 0.09 & 0.62 & -0.12 & 0.04 & 0.46 & 0.54 & 1.4 \\
\hline BDE-196 & 0.17 & 0.75 & 0.27 & -0.06 & -0.05 & 0.67 & 0.334 & 1.4 \\
\hline BDE-197 & 0.21 & 0.38 & 0.69 & -0.05 & 0 & 0.67 & 0.335 & 1.8 \\
\hline BDE-203 & 0.23 & 0.76 & 0.22 & 0.04 & -0.03 & 0.68 & 0.321 & 1.4 \\
\hline BDE-206 & -0.08 & 0.89 & -0.15 & 0.05 & 0.07 & 0.82 & 0.176 & 1.1 \\
\hline BDE-207 & -0.05 & 0.96 & 0.1 & 0.09 & 0.02 & 0.94 & 0.063 & 1 \\
\hline BDE-208 & -0.07 & 0.96 & 0.02 & 0.08 & 0.05 & 0.93 & 0.071 & 1 \\
\hline BDE-209 & -0.06 & 0.89 & -0.12 & 0.12 & 0.1 & 0.84 & 0.157 & 1.1 \\
\hline PCB-28 & 0.12 & -0.01 & -0.07 & 0.56 & 0.51 & 0.59 & 0.412 & 2.1 \\
\hline PCB-52 & 0.07 & 0 & 0.06 & 0.13 & 0.85 & 0.74 & 0.258 & 1.1 \\
\hline PCB-77 & 0.14 & 0.32 & 0 & -0.05 & 0.49 & 0.37 & 0.633 & 1.9 \\
\hline PCB-101 & 0.19 & -0.03 & 0.07 & 0.08 & 0.82 & 0.72 & 0.275 & 1.1 \\
\hline PCB-105 & 0.5 & -0.09 & 0.11 & 0.67 & 0.3 & 0.81 & 0.187 & 2.4 \\
\hline
\end{tabular}




\begin{tabular}{|c|c|c|c|c|c|c|c|c|}
\hline PCB-114 & 0.82 & -0.01 & -0.03 & 0.41 & 0.24 & 0.9 & 0.104 & 1.7 \\
\hline PCB-118 & 0.58 & -0.07 & 0.15 & 0.66 & 0.27 & 0.88 & 0.124 & 2.4 \\
\hline PCB-123 & 0.38 & -0.04 & -0.04 & 0.72 & 0.22 & 0.71 & 0.287 & 1.8 \\
\hline PCB-126 & 0.42 & -0.03 & 0.07 & 0.74 & 0.16 & 0.75 & 0.248 & 1.7 \\
\hline PCB-138 & 0.82 & -0.02 & 0.29 & 0.3 & 0.23 & 0.89 & 0.106 & 1.7 \\
\hline PCB-153 & 0.91 & 0 & 0.19 & 0.26 & 0.15 & 0.96 & 0.037 & 1.3 \\
\hline PCB-156 & 0.96 & 0.02 & 0 & 0.17 & 0.09 & 0.96 & 0.041 & 1.1 \\
\hline PCB-157 & 0.94 & 0 & -0.01 & 0.21 & 0.12 & 0.94 & 0.062 & 1.1 \\
\hline PCB-167 & 0.85 & 0.01 & 0.13 & 0.42 & 0.09 & 0.93 & 0.068 & 1.5 \\
\hline PCB-169 & 0.84 & 0.06 & 0.05 & 0.24 & 0.12 & 0.79 & 0.213 & 1.2 \\
\hline PCB-180 & 0.95 & 0.01 & 0.05 & 0.12 & 0 & 0.92 & 0.084 & 1 \\
\hline PCB-189 & 0.94 & 0.03 & 0 & 0.11 & -0.02 & 0.9 & 0.097 & 1 \\
\hline $1,2,3,6,7,8-\mathrm{HxCDD}$ & 0.49 & 0.12 & 0.03 & 0.4 & 0.01 & 0.41 & 0.588 & 2.1 \\
\hline 1,2,3,4,6,7,8-HpCDD & 0.07 & 0.08 & 0.22 & 0.63 & -0.1 & 0.47 & 0.528 & 1.4 \\
\hline OCDD & 0.23 & 0.11 & 0.36 & 0.59 & -0.2 & 0.59 & 0.415 & 2.4 \\
\hline 2,3,4,7,8-PeCDF & 0.46 & 0.14 & -0.07 & 0.47 & -0.1 & 0.47 & 0.533 & 2.3 \\
\hline $1,2,3,6,7,8-\mathrm{HxCDF}$ & 0.25 & 0.21 & 0 & 0.45 & 0.07 & 0.31 & 0.687 & 2.1 \\
\hline
\end{tabular}


Table S15 Associations between exposure to tertiles of five factors from principal component analysis and TH levels based on single- and multiple-factor models. All models were adjusted for maternal age, BMI, education level, parity, country of origin, smoking, diet, and breastfeeding duration.

Single-factor model $\beta(95 \% \mathrm{CI})$

Multi-factor model $\beta(95 \% \mathrm{CI})$

$\mathrm{TT}_{4}$

$\mathrm{TT}_{3}$

$\mathrm{TrT}_{3}$

$\mathrm{TT}_{4}$

$\mathrm{TT}_{3}$

$\operatorname{TrT}_{3}$

Factor $1^{\text {a }}$

$1 \quad$ Reference

Reference

Reference

Reference

Reference

Reference

2

$0.03(-0.10-0.15) \quad 0.04(-0.07-0.15) \quad 0.02(-0.20-0.24)$

$0.02(-0.10-0.14)$

$0.05(-0.06-0.16)$

$-0.01(-0.23-0.21)$

3

$0.07(-0.07-0.22) \quad 0.08(-0.05-0.20) \quad 0.00(-0.25-0.26)$

$0.08(-0.07-0.23)$

$0.09(-0.05-0.22)$

$-0.06(-0.32-0.21)$

Factor $2^{\mathrm{b}}$

$1 \quad$ Reference

Reference

Reference

Reference

Reference

Reference

2

$-0.01(-0.14-0.13) \quad-0.01(-0.12-0.10) \quad 0.05(-0.17-0.28)$

$0.01(-0.14-0.15)$

$-0.01(-0.13-0.12)$

$0.15(-0.10-0.40)$

3

$0.00(-0.13-0.14) \quad-0.04(-0.16-0.07) \quad-0.05(-0.28-0.18)$

$0.06(-0.08-0.20)$

$-0.01(-0.14-0.11) \quad 0.05(-0.20-0.30)$

Factor $3^{c}$

$1 \quad$ Reference

Reference

Reference

Reference

Reference

Reference
$-0.04(-0.16-0.09)$
$-0.02(-0.13-0.08)$
$-0.12(-0.33-0.09)$
$-0.07(-0.20-0.05)$
$-0.04(-0.16-0.07)$
$-0.22(-0.44-0.01)$
$-0.09(-0.20-0.01)$
$-0.21(-0.41-0.00) * \quad-0.16(-0.29--$
$-0.10(-0.22-0.01)$
$-0.30(-0.53--$
$0.00)^{*}$
$0.03)^{*}$
$0.07)^{*}$

$3-0.12(-0.24-$

Factor $4^{\mathrm{d}}$

Reference

Reference

Reference

Reference

Reference

Reference

$0.10(-0.03-0.24)$

$0.04(-0.07-0.16)$

$0.20(-0.02-0.43)$

$0.15(0.01-0.29)^{*} \quad 0.06(-0.06-0.18)$

$0.26(0.02-0.51)^{*}$ 


\begin{tabular}{lllllll}
3 & $-0.02(-0.14-0.10)$ & $-0.04(-0.15-0.07)$ & $0.08(-0.13-0.30)$ & $-0.01(-0.14-0.11)$ & $-0.03(-0.14-0.08)$ & $0.11(-0.11-0.33)$ \\
Factor $5^{\mathrm{e}}$ & & & & & \\
1 & Reference & Reference & Reference & Reference & Reference & Reference \\
2 & $-0.06(-0.19-0.06)$ & $-0.07(-0.18-0.04)$ & $-0.02(-0.24-0.20)$ & $-0.03(-0.16-0.10)$ & $-0.05(-0.16-0.06)$ & $0.02(-0.21-0.24)$ \\
$\mathrm{x} 3$ & $0.03(-0.09-0.16)$ & $0.00(-0.10-0.11)$ & $0.08(-0.14-0.29)$ & $0.01(-0.11-0.14)$ & $-0.01(-0.12-0.10)$ & $0.06(-0.16-0.28)$ \\
\hline
\end{tabular}


Figure S1 Thyroid hormone extraction procedures in human breast milk.

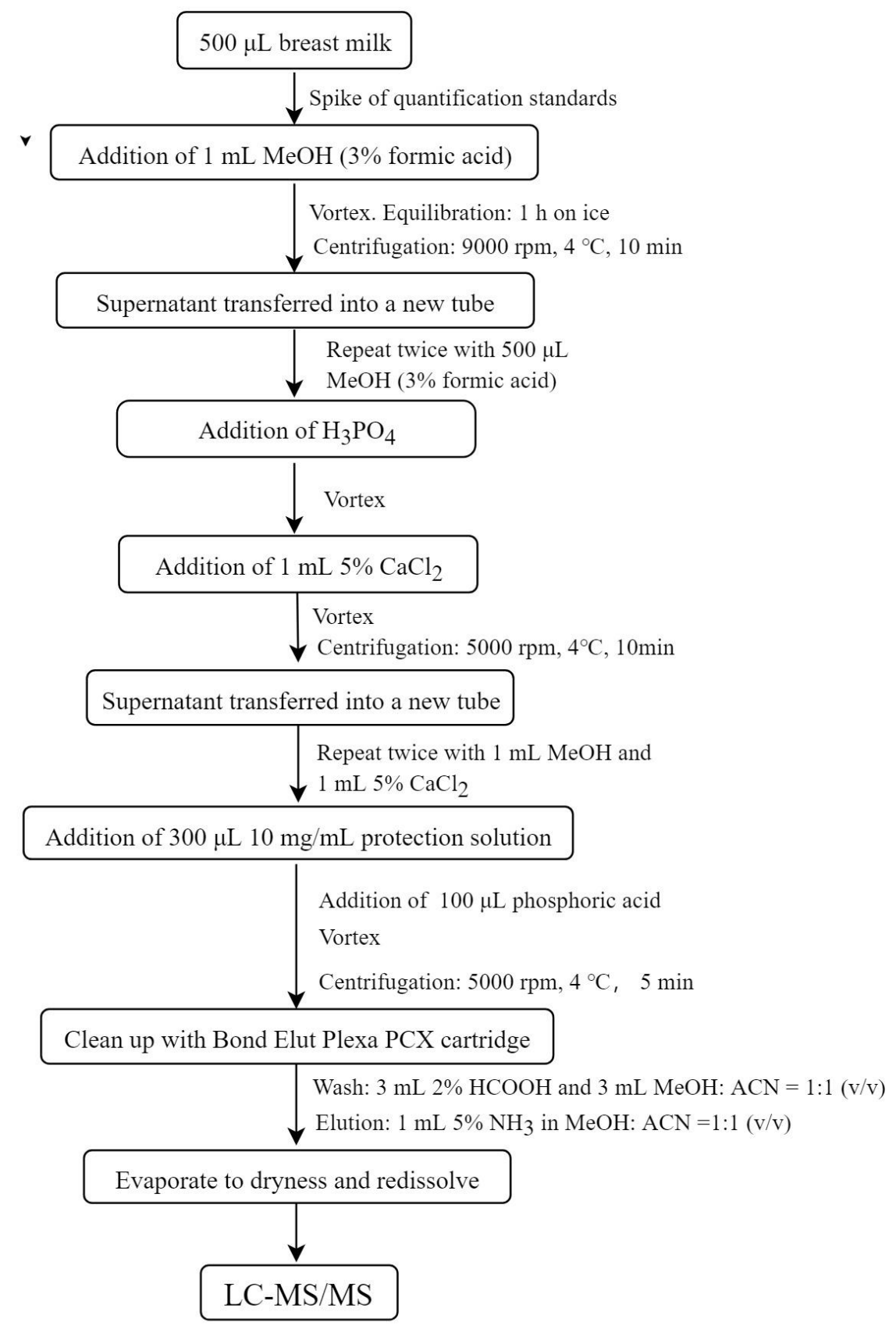


Figure S2 Representative MRM chromatograms of THs detected in human breast milk.

\section{Thyroid Hormones}
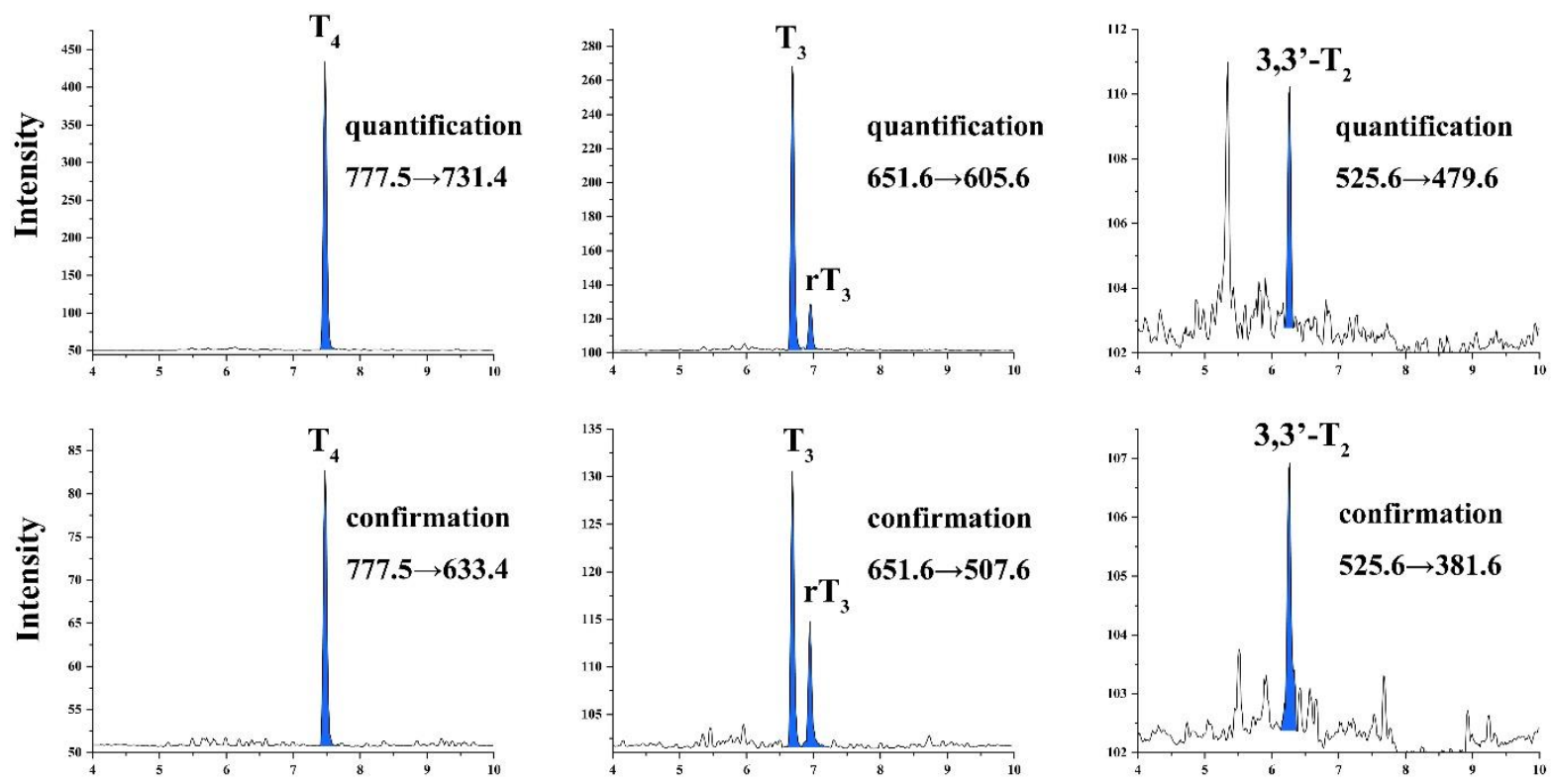

\section{Internal Standards}

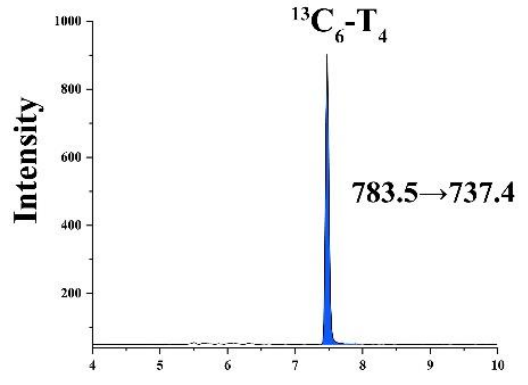

Time (min)

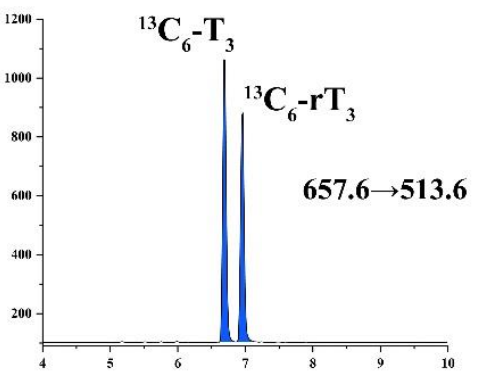

Time (min)

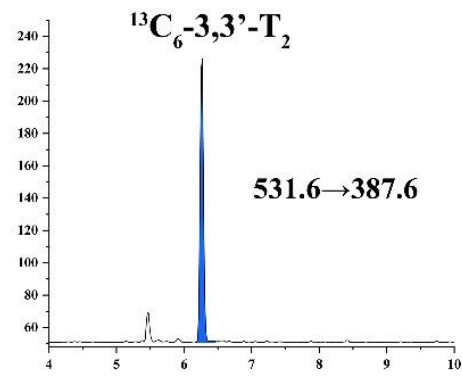

Time (min) 
Figure S3 Directed acyclic graph (DAG) illustrating relationships between milk POPs, THs, and covariates.

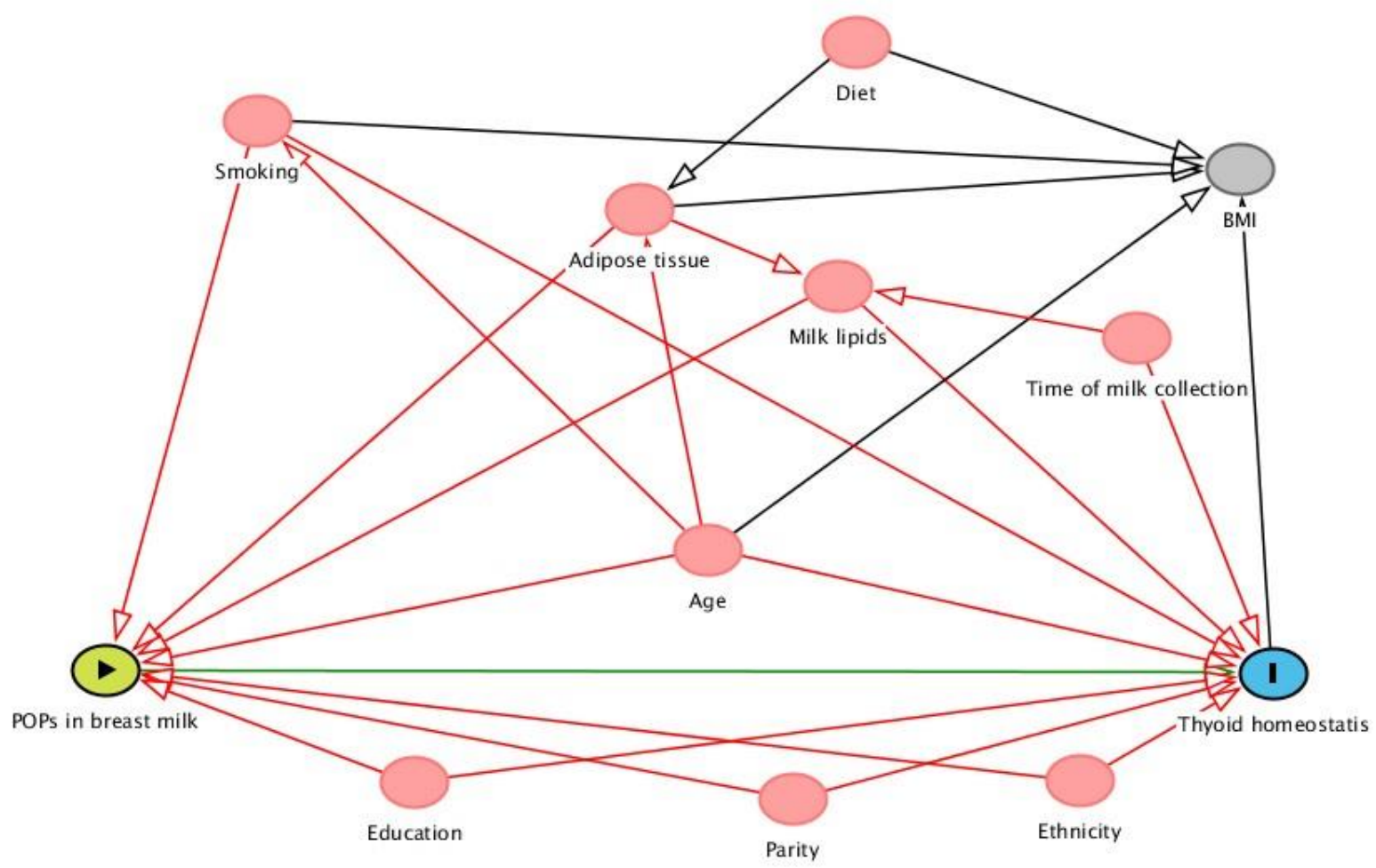


Figure S4 Spearman's rank correlation of biomarkers measured in human breast milk. THs were expressed in $\mathrm{ng} / \mathrm{mL}$ while POPs were expressed in $\mathrm{pg} / \mathrm{g}$ lw.

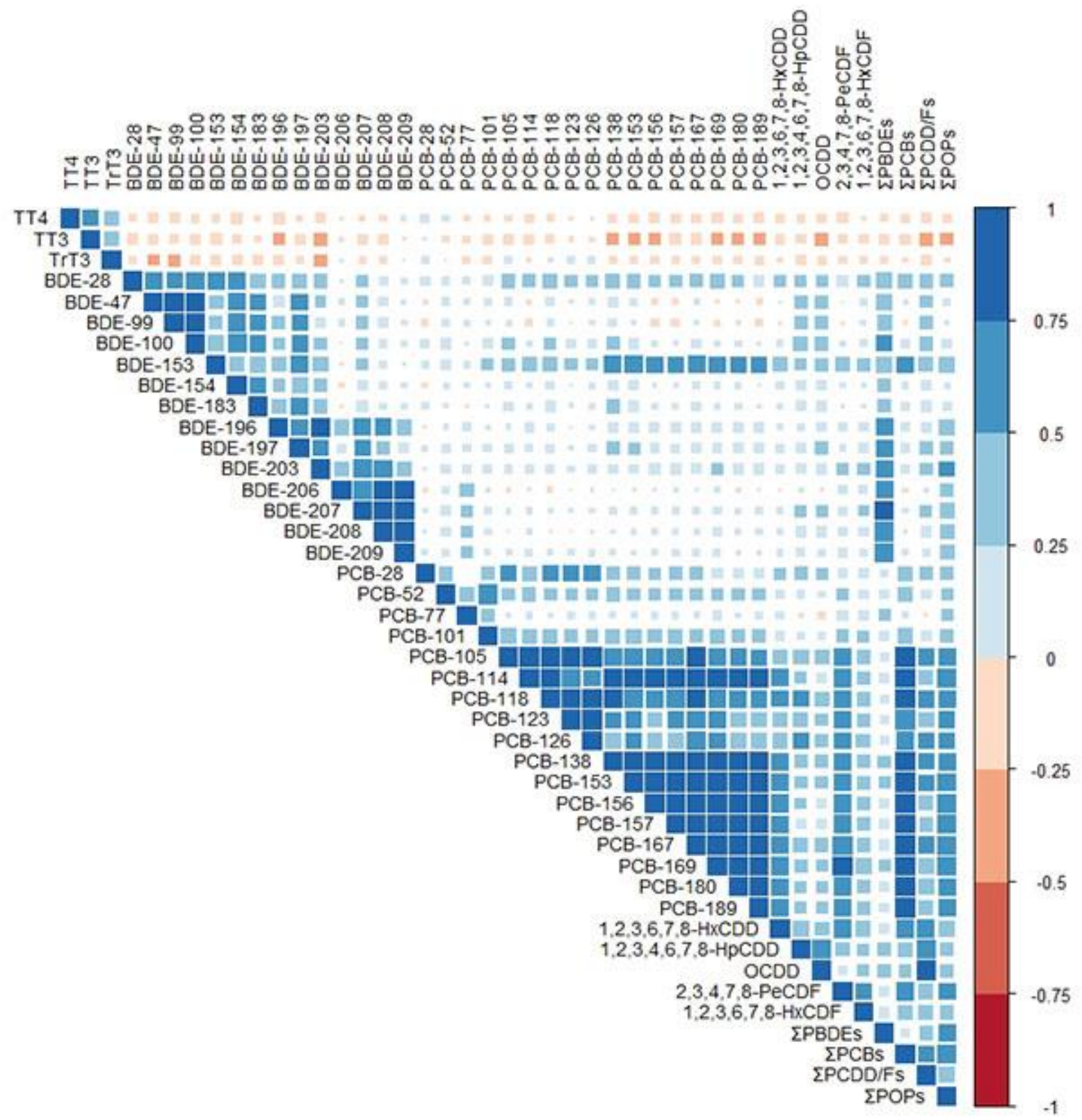


Figure S5 Hierarchical clustering of POPs based on concentrations in 99 serum samples. The figure depicts the hierarchical structure obtained from the correlation between compounds (method: complete linkage). The horizontal red line represents the manually selected cut-off for the number of clusters.

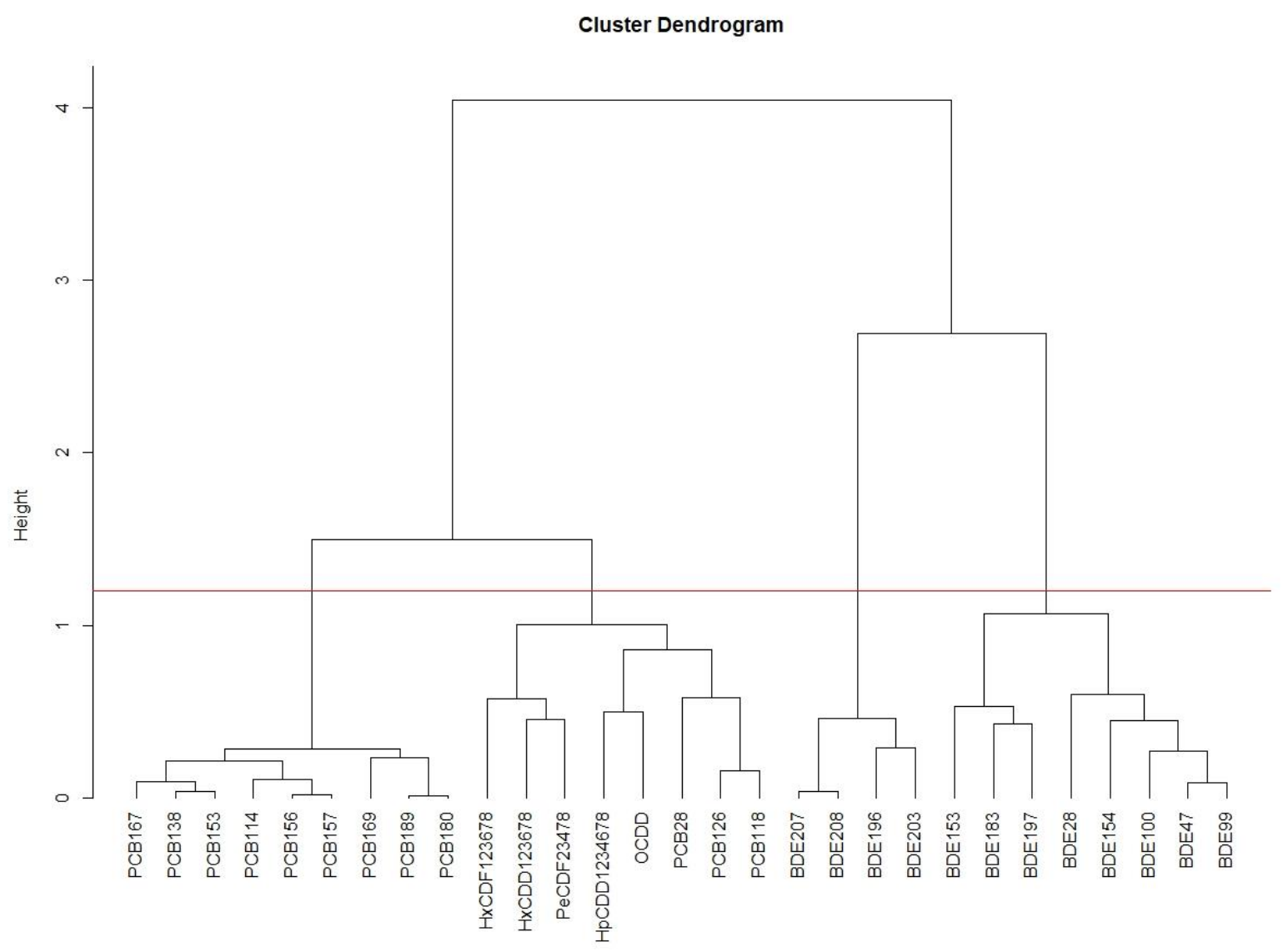




\section{References}

1. Li, Z. M.; Giesert, F.; Vogt-Weisenhorn, D.; Main, K. M.; Skakkebaek, N. E.; Kiviranta, H.; Toppari, J.; Feldt-Rasmussen, U.; Shen, H.; Schramm, K. W.; De Angelis, M., Determination of thyroid hormones in placenta using isotope-dilution liquid chromatography quadrupole time-of-flight mass spectrometry. J. Chromatogr. A 2017, 1534, 85-92.

2. Gordon, J. T.; Crutchfield, F. L.; Jennings, A. S.; Dratman, M. B., Preparation of lipid-free tissue extracts for chromatographic determination of thyroid hormones and metabolites. Arch. Biochem. Biophys. 1982, 216, (2), 407-415.

3. van Wassenaer, A. G.; Stulpt, M. R.; Valianpourt, F.; Tammlnga, P.; Stalpers, C. R.; de Randamie, J. S. E.; van Beusekom, C.; de Vijlder, J. J. M., The quantity of thyroid hormone in human milk is too low to influence plasma thyroid hormone levels in the very preterm infant. Clin. Endocrinol. (Oxf.) 2002, 56, 621-627.

4. Zhang, Q.; Lian, X.; Chai, X.; Bai, Y.; Dai, W., Relationship between maternal milk and serum thyroid hormones in patients with thyroid related diseases. Acta Academiae Medicinae Sinicae 2013, 35, (4), 427-431.

5. Lehmann, G. M.; LaKind, J. S.; Davis, M. H.; Hines, E. P.; Marchitti, S. A.; Alcala, C.; Lorber, M., Environmental chemicals in breast milk and formula: exposure and risk assessment implications. Environ. Health Perspect. 2018, 126, (9), 96001.

6. Chen, T.; Huang, M.; Li, J.; Li, J.; Shi, Z., Polybrominated diphenyl ethers and novel brominated flame retardants in human milk from the general population in Beijing, China: Occurrence, temporal trends, nursing infants' exposure and risk assessment. Sci. Total Environ. 2019, 689, 278-286. 
7. Zhang, L.; Yin, S.; Zhao, Y.; Shi, Z.; Li, J.; Wu, Y., Polybrominated diphenyl ethers and indicator polychlorinated biphenyls in human milk from China under the Stockholm Convention. Chemosphere 2017, 189, 32-38.

8. Chen, M.-W.; Castillo, B. A. A.; Lin, D.-Y.; Chao, H.-R.; Tayo, L. L.; Gou, Y.-Y.; Chen, F.-A.; Huang, K.-L., Levels of PCDD/Fs, PBDEs, and PBDD/Fs in breast milk from Southern Taiwan. Bull. Environ. Contam. Toxicol. 2018, 100, (3), 369-375.

9. Lee, S.; Kim, S.; Kim, E.; Lee, I. S.; Choi, G.; Kim, H. J.; Park, J.; Jae Lee, J.; Choi, S.; Young Kim, S.; Kim, S.; Kim, S.; Choi, K.; Moon, H. B., Polybrominated diphenyl ethers (PBDEs) in breast milk of Korea in 2011: current contamination, time course variation, influencing factors and health risks. Environ. Res. 2013, 126, 76-83.

10. Sudaryanto, A.; Kajiwara, N.; Takahashi, S.; Muawanah; Tanabe, S., Geographical distribution and accumulation features of PBDEs in human breast milk from Indonesia. Environ. Pollut. 2008, 151, (1), 130-8.

11. Eslami, B.; Koizumi, A.; Ohta, S.; Inoue, K.; Aozasa, O.; Harada, K.; Yoshinaga, T.; Date, C.; Fujii, S.; Fujimine, Y.; Hachiya, N.; Hirosawa, I.; Koda, S.; Kusaka, Y.; Murata, K.; Nakatsuka, H.; Omae, K.; Saito, N.; Shimbo, S.; Takenaka, K.; Takeshita, T.; Todoriki, H.; Wada, Y.; Watanabe, T.; Ikeda, M., Large-scale evaluation of the current level of polybrominated diphenyl ethers (PBDEs) in breast milk from 13 regions of Japan. Chemosphere 2006, 63, (4), 554-61.

12. Dimitriadou, L.; Malarvannan, G.; Covaci, A.; Iossifidou, E.; Tzafettas, J.; ZournatziKoiou, V.; Kalantzi, O. I., Levels and profiles of brominated and chlorinated contaminants in human breast milk from Thessaloniki, Greece. Sci. Total Environ. 2016, 539, 350-358. 
13. Croes, K.; Colles, A.; Koppen, G.; Govarts, E.; Bruckers, L.; Van de Mieroop, E.; Nelen, V.; Covaci, A.; Dirtu, A. C.; Thomsen, C.; Haug, L. S.; Becher, G.; Mampaey, M.; Schoeters, G.; Van Larebeke, N.; Baeyens, W., Persistent organic pollutants (POPs) in human milk: a biomonitoring study in rural areas of Flanders (Belgium). Chemosphere 2012, 89, (8), 988-94. 14. Bramwell, L.; Harrad, S.; Abou-Elwafa Abdallah, M.; Rauert, C.; Rose, M.; Fernandes, A.; Pless-Mulloli, T., Predictors of human PBDE body burdens for a UK cohort. Chemosphere 2017, 189, 186-197.

15. Lenters, V.; Iszatt, N.; Forns, J.; Cechova, E.; Kocan, A.; Legler, J.; Leonards, P.; Stigum, H.; Eggesbo, M., Early-life exposure to persistent organic pollutants (OCPs, PBDEs, PCBs, PFASs) and attention-deficit/hyperactivity disorder: A multi-pollutant analysis of a Norwegian birth cohort. Environ. Int. 2019, 125, 33-42.

16. Rawn, D. F. K.; Sadler, A. R.; Casey, V. A.; Breton, F.; Sun, W. F.; Arbuckle, T. E.; Fraser, W. D., Dioxins/furans and PCBs in Canadian human milk: 2008-2011. Sci. Total Environ. 2017, 595, 269-278.

17. Someya, M.; Ohtake, M.; Kunisue, T.; Subramanian, A.; Takahashi, S.; Chakraborty, P.; Ramachandran, R.; Tanabe, S., Persistent organic pollutants in breast milk of mothers residing around an open dumping site in Kolkata, India: specific dioxin-like PCB levels and fish as a potential source. Environ. Int. 2010, 36, (1), 27-35.

18. Mannetje, A.; Coakley, J.; Bridgen, P.; Brooks, C.; Harrad, S.; Smith, A. H.; Pearce, N.; Douwes, J., Current concentrations, temporal trends and determinants of persistent organic pollutants in breast milk of New Zealand women. Sci. Total Environ. 2013, 458-460, 399-407. 19. Iszatt, N.; Janssen, S.; Lenters, V.; Dahl, C.; Stigum, H.; Knight, R.; Mandal, S.; Peddada, 
S.; Gonzalez, A.; Midtvedt, T.; Eggesbo, M., Environmental toxicants in breast milk of Norwegian mothers and gut bacteria composition and metabolites in their infants at 1 month. Microbiome 2019, 7, (1), 34.

20. Chovancova, J.; Conka, K.; Kocan, A.; Sejakova, Z. S., PCDD, PCDF, PCB and PBDE concentrations in breast milk of mothers residing in selected areas of Slovakia. Chemosphere 2011, 83, (10), 1383-90.

21. Tajimi, M.; Watanabe, M.; Oki, I.; Ojima, T.; Nakamura, Y., PCDDs, PCDFs and Co-PCBs in human breast milk samples collected in Tokyo, Japan. Acta Paediatr. 2004, 93, (8), 10981102.

22. Schuhmacher, M.; Mari, M.; Nadal, M.; Domingo, J. L., Concentrations of dioxins and furans in breast milk of women living near a hazardous waste incinerator in Catalonia, Spain. Environ. Int. 2019, 125, 334-341. 